\title{
ON SARMANOV MIXED ERLANG RISKS IN INSURANCE APPLICATIONS
}

BY

\author{
ENKELEJD HASHORVA AND GILDAS RATOVOMIRIJA
}

\begin{abstract}
In this paper we consider an extension to the aggregation of the FGM mixed Erlang risks, proposed by Cossette et al. (2013 Insurance: Mathematics and Economics, 52, 560-572), in which we introduce the Sarmanov distribution to model the dependence structure. For our framework, we demonstrate that the aggregated risk belongs to the class of Erlang mixtures. Following results from S. C. K. Lee and X. S. Lin (2010 North American Actuarial Journal, 14(1) 107130), G. E. Willmot and X. S. Lin (2011 Applied Stochastic Models in Business and Industry, 27(1) 8-22), analytical expressions of the contribution of each individual risk to the economic capital for the entire portfolio are derived under both the TVaR and the covariance capital allocation principle. By analysing the commonly used dependence measures, we also show that the dependence structure is wide and flexible. Numerical examples and simulation studies illustrate the tractability of our approach.
\end{abstract}

\section{KEYWORDS}

Risk aggregation, Sarmanov distribution, mixed Erlang distribution, dependence measures, capital allocation.

\section{INTRODUCTION}

Analysis of aggregated risk is important for insurance business, it allows the insurers to assess and to monitor their risks through the risk management framework. In the classical framework of independent and identically distributed risks, explicit analytical formulas for quantities of interest including Value-atRisk (VaR), Tail Value-at-Risk (TVaR) or Stop-loss premium formula for the aggregated risk can be derived explicitly for few tractable cases. For instance Willmot and Lin (2011), Lee and Lin (2010, 2012) and Cossette et al. (2013) have shown that this is the case if we choose the mixed Erlang distribution as a model for claim sizes. One reason for the tractability of the mixed Erlang distribution is the fact that the convolution of such risks is again mixed Erlang, see Klugman et al. (2008). 
Since insurance data clearly shows that insurance risks are commonly dependent, in order to be able to get closed-form formulas for quantities of interest, an important task is the adequate choice of the dependence structure between the risks. Even for the simple case of the dependence specified by a log-normal framework with stochastic volatility, as shown in the recent contributions (Embrechts et al., 2014; Hashorva and Kortschak, 2014; Hashorva and Li, 2015) only asymptotic results can be derived.

With motivation from Cossette et al. (2013) where the aggregation of FGM mixed Erlang risks is considered, in this contribution we shall investigate the Sarmanov mixed Erlang risks. The Sarmanov distribution includes the FGM distribution as a special case. One key advantage of the Sarmanov distribution is its flexibility; it also allows to model highly dependent risks, see e.g., Bairamov et al. (2001) and Lee (1996). The aim of this paper is to provide analytical results and properties of the aggregated dependent risks with mixed Erlang marginals by using the Sarmanov distribution as a model for the dependence structure. This model is promising in risk aggregation practice as it satisfies the four desirable properties of a multivariate parametric model mentioned in Joe (1997) p. 84, namely the interpretability property, the closure property, the flexibility and the wideness of the range of dependence, and the representation of the distribution function (df) and the probability density function (pdf) in analytical form.

The paper is organised as follows. In Section 2, we describe the background of the Sarmanov mixed Erlang distribution by exploring some definitions and properties of the Sarmanov distribution as a model for the dependence structure and the mixed Erlang distribution with a common scale parameter as a model for claim size distribution in insurance. In Section 3, we demonstrate that the distribution of the aggregated risk belongs to the class of Erlang mixtures; numerical illustrations and simulation studies are performed to show the robustness of the results. In Section 4, we derive explicit expressions for the allocated capital to each individual risk $X_{i}, i=1,2$ under the TVaR and the covariance capital allocation rules. We present some useful results and properties of the mixed Erlang distribution in Section 5. In Section 6, an extension of the results in the bivariate case to the multivariate framework is presented with numerical examples. All the proofs are relegated to Section 7. In the Appendix, the flexibility and the wideness of the dependence range of Sarmanov mixed Erlang distributions are discussed by calculating commonly used dependence measures, namely Pearson's correlation coefficient, Sperman's rho and Kendall's tau.

\section{PRELIMINARIES}

\subsection{Sarmanov distribution}

The Sarmanov distribution introduced in Sarmanov (1966) has proved valuable in numerous insurance applications. For instance Hernández-Bastida and Fernández-Sánchez (2012) used the multivariate Sarmanov distribution to model the dependence structure between risk profiles for the calculation 
of Bayes premiums in the collective risk model. The contribution (Sarabia and Gómez-Déniz, 2011) fitted multivariate insurance count data using the Sarmanov distribution with Poisson-Beta marginals. As shown in Yang and Hashorva (2013) and Yang and Wang (2013), the Sarmanov distribution allows for tractable asymptotic formulas in the context of ruin probabilities. Referring to Sarmanov (1966), a bivariate risk $\left(X_{1}, X_{2}\right)$ has the Sarmanov distribution with joint pdf $h$ given by

$$
h\left(x_{1}, x_{2}\right)=f_{1}\left(x_{1}\right) f_{2}\left(x_{2}\right)\left(1+\alpha_{12} \phi_{1}\left(x_{1}\right) \phi_{2}\left(x_{2}\right)\right), \quad \alpha_{12} \in \mathbb{R},
$$

where $f_{i}$ is the pdf of $X_{i}, i=1,2$, and $\phi_{1}, \phi_{2}$ are two kernel functions, which are assumed to be bounded and non-constant such that

$$
\mathbb{E}\left(\phi_{1}\left(X_{1}\right)\right)=\mathbb{E}\left(\phi_{2}\left(X_{2}\right)\right)=0, \quad 1+\alpha_{12} \phi_{1}\left(x_{1}\right) \phi_{2}\left(x_{2}\right) \geqslant 0
$$

is valid. If $\phi_{i}\left(x_{i}\right)=1-2 F_{i}\left(x_{i}\right)$ with $F_{i}$ the df of $X_{i}$, then $h$ is the joint pdf of the FGM distribution introduced by Morgenstern (1956) for Cauchy marginals and developed by Gumbel (1960) for exponential margins and generalized by Farlie (1960). Lee (1996) proposed some general methods for finding the kernel function $\phi_{i}\left(x_{i}\right)$ with different types of marginals. Yang and Hashorva (2013) considered $\phi_{i}\left(x_{i}\right)=g_{i}\left(x_{i}\right)-\mathbb{E}\left(g_{i}\left(X_{i}\right)\right)$. When $g_{i}\left(x_{i}\right)=e^{-x_{i}}$ the corresponding kernel function coincides with the one explored by Lee (1996) for marginal distributions with support in $[0, \infty)$. We have

$$
\phi_{i}\left(x_{i}\right)=e^{-x_{i}}-\mathbb{E}\left(e^{-X_{i}}\right)=e^{-x_{i}}-\mathcal{L}_{i}(1),
$$

where $\mathcal{L}_{i}(t)=\mathbb{E}\left(e^{-t X_{i}}\right), t>0$ is the Laplace transform of $X_{i}$. In the rest of the paper, we set

$$
\mathcal{L}_{i}:=\mathcal{L}_{i}(1), \quad \mathcal{L}_{i}^{\prime}:=\mathcal{L}_{i}^{\prime}(1) .
$$

The joint pdf $h$ is thus given by

$$
\begin{aligned}
h\left(x_{1}, x_{2}\right) & =f_{1}\left(x_{1}\right) f_{2}\left(x_{2}\right)\left((1+\gamma)+\alpha_{12}\left(e^{-x_{1}-x_{2}}-e^{-x_{1}} \mathcal{L}_{2}-e^{-x_{2}} \mathcal{L}_{1}\right)\right), \\
\gamma & =\alpha_{12} \mathcal{L}_{1} \mathcal{L}_{2} .
\end{aligned}
$$

Remarks 2.1. If $\left(X_{1}, X_{2}\right)$ has a Sarmanov distribution with kernel functions given in (2.3), additionally if $X_{i}, i=1,2$ follows a mixture of Gamma distributions where the mixture components share the same scale parameter $\beta_{i} \in(0, \infty)$, then the joint df of $\left(X_{1}, X_{2}\right)$ follows easily from integrating the $p d f$ in (2.4). Specifically, we have for $H$ the joint df of $\left(X_{1}, X_{2}\right)$

$$
\begin{aligned}
H\left(x_{1}, x_{2}\right)= & (1+\gamma) F_{1}\left(x_{1}, \beta_{1}\right) F_{2}\left(x_{2}, \beta_{2}\right)+\gamma F_{1}\left(x_{1}, \beta_{1}+1\right) F_{2}\left(x_{2}, \beta_{2}+1\right) \\
& -\gamma F_{1}\left(x_{1}, \beta_{1}+1\right) F_{2}\left(x_{2}, \beta_{2}\right)-\gamma F_{1}\left(x_{1}, \beta_{1}\right) F_{2}\left(x_{2}, \beta_{2}+1\right),
\end{aligned}
$$

where $F_{i}\left(x_{i}, \beta_{i}\right)=\sum_{k=1}^{\infty} q_{k} W_{k}\left(x_{i}, \beta_{i}\right), i=1,2$ with $W_{k}\left(x_{i}, \beta_{i}\right)$ is the $d f$ of the Gamma distribution with scale parameter $\beta_{i}$ and shape parameter $k \in(0, \infty)$ and $q_{k}$ is the mixing weight such that $\sum_{k=1}^{\infty} q_{k}=1$. 
Compared to the FGM distribution which has $[-1 / 3,1 / 3]$ as the range of Pearson's correlation coefficient $\rho_{12}$ the Sarmanov distribution has a wider range of $\rho_{12}$, which is useful in the aggregation of strongly dependent insurance risks. For the Sarmanov case we have the explicit formula for $\rho_{12}$, namely

$$
\rho_{12}\left(X_{1}, X_{2}\right)=\frac{\alpha_{12} v_{1} \nu_{2}}{\sigma_{1} \sigma_{2}}, \quad v_{i}=\mathbb{E}\left(X_{i} \phi_{i}\left(X_{i}\right)\right), \quad \sigma_{i}=\sqrt{\operatorname{Var}\left(X_{i}\right)}, \quad i=1,2 .
$$

In the particular case that the kernels are given by (2.3), for two positive Sarmanov risks with finite variances the range of $\alpha_{12}$ is (see Lee, 1996)

$$
\frac{-1}{\max \left\{\mathcal{L}_{1} \mathcal{L}_{2},\left(1-\mathcal{L}_{1}\right)\left(1-\mathcal{L}_{2}\right)\right\}} \leqslant \alpha_{12} \leqslant \frac{1}{\max \left\{\mathcal{L}_{1}\left(1-\mathcal{L}_{2}\right),\left(1-\mathcal{L}_{1}\right) \mathcal{L}_{2}\right\}},
$$

where $v_{i}=-\mathcal{L}_{i}^{\prime}-\mathcal{L}_{i} \mu_{i}$ and $\mu_{i}=\mathbb{E}\left(X_{i}\right), i=1$, 2. Lee (1996) extended the Sarmanov distribution to the multivariate case by defining the joint pdf $h$ of $\left(X_{1}, \ldots, X_{n}\right)$ as

$$
h(\boldsymbol{x})=\prod_{i=1}^{n} f_{i}\left(x_{i}\right)\left(1+R_{\phi_{1}, \ldots, \phi_{n}, \Omega_{n}}(\boldsymbol{x})\right), \quad \boldsymbol{x}:=\left(x_{1}, \ldots, x_{n}\right),
$$

where

$$
\begin{aligned}
R_{\phi_{1}, \ldots, \phi_{n}, \Omega_{n}}(\boldsymbol{x})= & 1+\sum_{j_{1}<}^{n-1} \sum_{j_{2}}^{n} \alpha_{j_{1}, j_{2}} \phi_{j_{1}}\left(x_{j_{1}}\right) \phi_{j_{2}}\left(x_{j_{2}}\right) \\
& +\sum_{j_{1}<}^{n-2} \sum_{j_{2}<}^{n-1} \sum_{j_{3}}^{n} \alpha_{j_{1}, j_{2}, j_{3}} \phi_{j_{1}}\left(x_{j_{1}}\right) \phi_{j_{2}}\left(x_{j_{2}}\right) \phi_{j_{3}}\left(x_{j_{3}}\right)+\cdots+\alpha_{1,2, \ldots, n} \\
& \times \prod_{i=1}^{n} \phi_{i}\left(x_{i}\right)
\end{aligned}
$$

such that

$$
1+R_{\phi_{1}, \ldots, \phi_{n}, \Omega_{n}}(\boldsymbol{x}) \geqslant 0
$$

is fulfilled for all $x_{i} \in \mathbb{R}$ with $\Omega_{n}=\left\{\alpha_{j_{1}, j_{2}}, \alpha_{j_{1}, j_{2}, j_{3}}, \ldots, \alpha_{1,2, \ldots, n}\right\} \in \mathbb{R}$. If the kernel functions are specified by $(2.3)$, then $h$ is given by $\left(\operatorname{set} \Delta\left(x_{i}\right):=e^{-x_{i}}-\mathcal{L}_{i}\right)$

$$
\begin{aligned}
h(\boldsymbol{x})= & \prod_{i=1}^{n} f_{i}\left(x_{i}\right)\left(1+\sum_{j_{1}<}^{n-1} \sum_{j_{2}}^{n} \alpha_{j_{1}, j_{2}} \Delta\left(x_{j_{1}}\right) \Delta\left(x_{j_{2}}\right)\right. \\
& \left.+\sum_{j_{1}<}^{n-2} \sum_{j_{2}<}^{n-1} \sum_{j_{3}}^{n} \alpha_{j_{1}, j_{2}, j_{3}} \Delta\left(x_{j_{1}}\right) \Delta\left(x_{j_{2}}\right) \Delta\left(x_{j_{3}}\right)+\cdots+\alpha_{1,2, \ldots, n} \prod_{i=1}^{n} \Delta\left(x_{i}\right)\right) .
\end{aligned}
$$




\subsection{Mixed Erlang claim sizes}

These last decades, modeling claim size in insurance with the mixed Erlang distribution with a common scale parameter has been well developed. In risk theory, Dickson and Willmot (2005) and Dickson (2008) have explored an analytical form of the finite time ruin probability, using the mixed Erlang distribution as a claim size model. Recently, using the EM algorithm Lee and Lin (2010) have fitted some common parametric distributions and catastrophic loss data in the United States with the mixed Erlang distribution. Moreover, Lee and Lin (2012) have developed the multivariate mixed Erlang distribution to overcome some drawbacks of the copula approach. Furthermore, Cossette et al. (2013) have introduced a risk aggregation in the multivariate setup with mixed Erlang marginals and the FGM copula to capture the dependence structure. As its name indicates, the mixed Erlang distribution is constructed from the Erlang distribution which has the pdf

$$
w_{k}(x, \beta)=\frac{\beta^{k} x^{k-1} e^{-\beta x}}{(k-1) !}, \quad x>0,
$$

where $k \in \mathbb{N}^{*}$ is the shape parameter and $\beta>0$ is the scale parameter. Hence, the pdf of the mixed Erlang distribution is defined as

$$
f(x, \beta, Q)=\sum_{k=1}^{\infty} q_{k} w_{k}(x, \beta),
$$

where $Q=\left(q_{1}, q_{2}, \ldots\right)$ is a vector of non-negative weights satisfying $\sum_{k=1}^{\infty} q_{k}=$ 1. In the following we write $X \sim \operatorname{ME}(\beta, Q)$ if $X$ has pdf given by (2.12). By integrating the pdf in (2.12) the df $F$ corresponding to $f$ is given by

$$
F(x, \beta, Q)=1-e^{-\beta x} \sum_{k=1}^{\infty} q_{k} \sum_{j=0}^{k-1} \frac{(\beta x)^{j}}{j !} .
$$

As discussed in Lee and Lin (2010, 2012), Willmot and Lin (2011) and Cossette et al. (2013) one of the important advantages of employing the mixed Erlang distribution in insurance loss modeling is the fact that many useful risk related quantities, such as moments and mean excess function can be calculated explicitly by simple formulas. For instance, the quantile function (or VaR) of the mixed Erlang distribution can be easily obtained given the tractable form of the df. From (2.13), at a confidence level $p \in(0,1)$, the VaR of $X$, denoted by $x_{p}$, is the solution of

$$
e^{-\beta x_{p}} \sum_{k=1}^{\infty} q_{k} \sum_{j=0}^{k-1} \frac{\left(\beta x_{p}\right)^{j}}{j !}=1-p,
$$


which can be solved numerically. Further, since for the mean excess function of $X$, we have (see Willmot and Lin (2011), p. 7)

$$
\mathbb{E}((X-d) \mid X>d)=\frac{\sum_{k=0}^{\infty} Q_{k}^{*} \frac{(\beta d)^{k}}{k !}}{\beta \sum_{j=1}^{\infty} Q_{j} \frac{(\beta d)^{j-1}}{(j-1) !}}, \quad d>0,
$$

where $Q_{k}^{*}=\sum_{j=k+1}^{\infty} Q_{j}$ with $Q_{j}=\frac{\sum_{k=j}^{\infty} q_{k}}{\sum_{k=1}^{\infty} k q_{k}}$, then the TVaR of $X$ at a confidence level $p \in(0,1)$ is given by the following explicit formula

$$
T \operatorname{Va} R_{X}(p)=\frac{\sum_{k=0}^{\infty} Q_{k}^{*} \frac{\left(\beta x_{p}\right)^{k}}{k !}}{\beta \sum_{j=1}^{\infty} Q_{j} \frac{\left(\beta x_{p}\right)^{j-1}}{(j-1) !}}+x_{p} .
$$

Remark that above we assume that $\mathbb{E}(X)=\sum_{k=1}^{\infty} k q_{k}$ is finite. Additionally, the mixed Erlang distribution is a tractable marginal distribution for the Sarmanov distribution. Next we present a result for the 2-dimensional setup, see Section 6 for the same results in higher dimensions.

\section{Aggregation of SARMAnOv Mixed ERlang RiskS}

Let $\left(X_{1}, X_{2}\right)$ have a bivariate Sarmanov risk with kernel functions $\phi_{i}(x)=e^{-x_{i}}-$ $\mathcal{L}_{i}$ for $i=1,2$. We shall assume that both $X_{1}$ and $X_{2}$ follow a mixed Erlang distribution, i.e.,

$$
X_{i} \sim \operatorname{ME}\left(\beta_{i}, Q_{i}\right), \quad i=1,2,
$$

where $\beta_{i}$ is the scale parameter, $Q_{i}=\left(q_{i, 1}, q_{i, 2}, \ldots\right)$ denotes the mixing probabilities. The joint distribution of the random vector $\left(X_{1}, X_{2}\right)$ will be referred to as a bivariate Sarmanov mixed Erlang (SmE) distribution and we shall abbreviate this as $\left(X_{1}, X_{2}\right) \sim S M E_{2}\left(\boldsymbol{\beta}, Q_{1}, Q_{2}\right)$ where $\boldsymbol{\beta}=\left(\beta_{1}, \beta_{2}\right)$. The dependence structure of the bivariate random vector $\left(X_{1}, X_{2}\right)$ can be analysed by calculating commonly used dependence measures such as Pearson's correlation coefficient or Kendall's tau, see Appendix A. For given vectors of the mixing probabilities $V_{i}=\left(v_{i 1}, v_{i 2}, \ldots\right), i=1,2$ we define in the following $\pi_{1}\left\{L_{1}, V_{2}\right\}=0$ and for $k>1$

$$
\pi_{k}\left\{V_{1}, V_{2}\right\}=\sum_{j=1}^{k-1} v_{1, j} v_{2, k-j}
$$

The main result in this section is the derivation of the distribution of the aggregated risk $S_{2}=X_{1}+X_{2}$.

Proposition 3.1. If $\left(X_{1}, X_{2}\right) \sim \operatorname{SME}_{2}\left(\boldsymbol{\beta}, Q_{1}, Q_{2}\right)$ with $\beta_{1} \leqslant \beta_{2}$, then $S_{2} \sim$ $M E\left(\beta_{2}+1, P\right)$ where the mixing weights $p_{k}$ are given by (set $\gamma:=\alpha_{12} \mathcal{L}_{1} \mathcal{L}_{2}$, 
TABLE 3.1

Central moments of $X_{1}$ And $X_{2}$.

\begin{tabular}{ccccc}
\hline \hline & Mean & Variance & Skewness & Kurtosis \\
\hline$X_{1}$ & 2.33 & 4.44 & 1.38 & 5.49 \\
$X_{2}$ & 2.11 & 3.10 & 1.49 & 6.12 \\
\hline \hline
\end{tabular}

$\left.\overline{\beta_{i}}:=\beta_{i} /\left(\beta_{i}+1\right)\right)$

$$
\begin{aligned}
p_{k}= & (1+\gamma) \pi_{k}\left\{\Psi_{1}\left(Q_{1}\right), \Psi_{2}\left(Q_{2}\right)\right\}+\gamma \pi_{k}\left\{\Psi_{1}\left(\Theta_{1}\right), \Psi_{2}\left(\Theta_{2}\right)\right\} \\
& -\gamma \pi_{k}\left\{\Psi_{1}\left(\Theta_{1}\right), \Psi_{2}\left(Q_{2}\right)\right\}-\gamma \pi_{k}\left\{\Psi_{1}\left(Q_{1}\right), \Psi_{2}\left(\Theta_{2}\right)\right\},
\end{aligned}
$$

where for $i=1,2$ the components of $\Theta_{i}=\left(\theta_{i, 1}, \theta_{i, 2}, \ldots\right)$ are defined by $\theta_{i, k}=\frac{q_{i, k} \overline{\beta_{i}}{ }^{k}}{\sum_{j=1}^{\infty} q_{i, j} \overline{\beta_{i}}}$, whereas the components of $\Psi_{i}\left(Q_{i}\right)=\left(\psi_{i, 1}, \psi_{i, 2}, \ldots\right)$ are $\psi_{i, k}=\sum_{j=1}^{k} q_{i, j}\left(\begin{array}{c}k-1 \\ j-1\end{array}\right)\left(\frac{\beta_{i}}{\beta_{2}+1}\right)^{j}\left(1-\frac{\beta_{i}}{\beta_{2}+1}\right)^{k-j}$.

Example 3.2. As an illustration, let

$$
\begin{gathered}
\left(X_{1}, X_{2}\right) \sim S M E_{2}\left(\boldsymbol{\beta}=\left(\begin{array}{c}
0.9 \\
0.95
\end{array}\right) ; Q_{1}=(0.4,0.2,0.3,0.1) ;\right. \\
\left.Q_{2}=(0.3,0.5,0.1,0.1) ; \alpha_{12}=2.87\right) .
\end{gathered}
$$

According to (2.12), one can write the pdf of $X_{1}$ and $X_{2}$ as follows

$$
\begin{aligned}
& f_{1}\left(x_{1}\right)=0.4 w_{1}\left(x_{1}, 0.9\right)+0.2 w_{2}\left(x_{1}, 0.9\right)+0.3 w_{3}\left(x_{1}, 0.9\right)+0.1 w_{4}\left(x_{1}, 0.9\right) \\
& f_{2}\left(x_{2}\right)=0.3 w_{1}\left(x_{2}, 0.95\right)+0.5 w_{2}\left(x_{2}, 0.95\right)+0.1 w_{3}\left(x_{2}, 0.95\right)+0.1 w_{4}\left(x_{2}, 0.95\right) .
\end{aligned}
$$

Following (2.4), the joint density of $\left(X_{1}, X_{2}\right)$ is given by

$$
h\left(x_{1}, x_{2}\right)=f_{1}\left(x_{1}\right) f_{2}\left(x_{2}\right)\left(1.22+2.87 e^{-x_{1}-x_{2}}-0.81 e^{-x_{1}}-0.78 e^{-x_{2}}\right) .
$$

Table 3.1 above presents the central moments of the marginals.

It follows that the distribution of $S_{2}$ is a mixed Erlang distribution with scale parameter $\beta_{S_{2}}=1.95$ and mixing probabilities partially shown in 3.2. We notice that the higher the value of $k$ is, the smaller the value of $p_{k}$.

In order to validate our results, SmE risks have been simulated (see in Appendix $B$ the details about the simulation algorithm). In this respect, analytical and simulated results on the aggregated risk $S_{2}=X_{1}+X_{2}$ are presented and analysed. As displayed in Table 3.3, based on the VaR and TVaR risk measures the comparison of the exact and the simulated values shows that our results are robust for different values of the tolerance level $p$. Furthermore, it can be seen that $\mathrm{VaR}$ is more sensitive to the change of the tolerance level than TVaR. Similarly, by 
TABLE 3.2

MIXING PROBABILITIES OF THE DISTRIBUTION OF $S_{2}=X_{1}+X_{2}$, WITH SCALE PARAMETER $\beta_{S_{2}}=1.95$.

\begin{tabular}{cccccccccc}
\hline \hline$k$ & $p_{k}$ & $k$ & $p_{k}$ & $k$ & $p_{k}$ & $k$ & $p_{k}$ & $k$ & $p_{k}$ \\
\hline 1 & 0.0000 & 11 & 0.0664 & 21 & 0.0046 & 31 & $8.963 \mathrm{E}-05$ & 41 & $9.294 \mathrm{E}-07$ \\
2 & 0.0675 & 12 & 0.0564 & 22 & 0.0033 & 32 & $5.803 \mathrm{E}-05$ & 42 & $5.751 \mathrm{E}-07$ \\
3 & 0.0839 & 13 & 0.0465 & 23 & 0.0023 & 33 & $3.737 \mathrm{E}-05$ & 43 & $3.547 \mathrm{E}-07$ \\
4 & 0.0645 & 14 & 0.0373 & 24 & 0.0016 & 34 & $2.393 \mathrm{E}-05$ & 44 & $2.180 \mathrm{E}-07$ \\
5 & 0.0700 & 15 & 0.0292 & 25 & 0.0011 & 35 & $1.525 \mathrm{E}-05$ & 45 & $1.336 \mathrm{E}-07$ \\
6 & 0.0740 & 16 & 0.0223 & 26 & 0.0007 & 36 & $9.668 \mathrm{E}-06$ & 46 & $8.159 \mathrm{E}-08$ \\
7 & 0.0811 & 17 & 0.0168 & 27 & 0.0005 & 37 & $6.103 \mathrm{E}-06$ & 47 & $4.970 \mathrm{E}-08$ \\
8 & 0.0840 & 18 & 0.0125 & 28 & 0.0003 & 38 & $3.835 \mathrm{E}-06$ & 48 & $3.02 \mathrm{E}-08$ \\
9 & 0.0816 & 19 & 0.0091 & 29 & 0.0002 & 39 & $2.400 \mathrm{E}-06$ & 49 & $1.828 \mathrm{E}-08$ \\
10 & 0.0753 & 20 & 0.0065 & 30 & 0.0001 & 40 & $1.496 \mathrm{E}-06$ & 50 & $1.105 \mathrm{E}-08$ \\
\hline \hline
\end{tabular}

TABLE 3.3

EXACT AND SIMULATED VALUES OF VAR AND TVAR OF $S_{2}=X_{1}+X_{2}$.

\begin{tabular}{|c|c|c|c|c|c|c|}
\hline \multirow[b]{2}{*}{$p$} & \multicolumn{2}{|c|}{ Analytical Formula } & \multicolumn{2}{|c|}{ Simulated } & \multicolumn{2}{|c|}{ Percentage Difference (\%) } \\
\hline & $\operatorname{VaR}_{S_{2}}(p)$ & $T V a R_{S_{2}}(p)$ & $\operatorname{VaR}_{S_{2}}(p)$ & $T V a R_{S_{2}}(p)$ & $\operatorname{VaR}_{S_{2}}(p)$ & $T V a R_{S_{2}}(p)$ \\
\hline $90.00 \%$ & 8.26 & 10.24 & 8.22 & 10.21 & 0.49 & 0.29 \\
\hline $92.50 \%$ & 8.88 & 10.80 & 8.86 & 10.77 & 0.23 & 0.28 \\
\hline $95.00 \%$ & 9.71 & 11.56 & 9.66 & 11.53 & 0.52 & 0.26 \\
\hline $97.50 \%$ & 11.05 & 12.82 & 10.98 & 12.82 & 0.64 & 0.00 \\
\hline $99.00 \%$ & 12.71 & 14.41 & 12.79 & 14.46 & -0.63 & -0.35 \\
\hline $99.50 \%$ & 13.92 & 15.56 & 13.87 & 15.43 & 0.36 & 0.84 \\
\hline $99.90 \%$ & 16.57 & 18.13 & 16.61 & 17.86 & -0.24 & 1.51 \\
\hline $99.99 \%$ & 20.15 & 21.62 & 19.42 & 20.79 & 3.62 & 3.84 \\
\hline
\end{tabular}

changing the level of the dependence between marginals which is described by $\alpha_{12}$ and for a tolerance level of $99 \%$, the comparison of the exact and the simulated values of VaR and TVaR is displayed in Table 3.4. Note in passing that the maximum attainable value of $\alpha_{12}$, in our example, is 4.87 while the minimum is -1.91 .

\section{CApital Allocation}

In this section, we derive analytical expressions for the amount of capital allocated to each individual risk under the TVaR and the covariance principles. Evaluating the economic capital for the entire portfolio that an insurance company needs to absorb large unexpected losses is of importance in enterprise risk management. In this respect, the so-called capital allocation consists in determining the contribution of each individual risk to the aggregate economic capital. This allows the insurance company to identify and to monitor efficiently 
TABLE 3.4

DEPENDENCE LEVEL AND SENSITIVENESS OF RISK MEASURES.

\begin{tabular}{|c|c|c|c|c|c|c|}
\hline \multirow[b]{2}{*}{$\alpha_{12}$} & \multicolumn{2}{|c|}{ Analytical formula } & \multicolumn{2}{|c|}{ Simulated } & \multicolumn{2}{|c|}{ Percentage difference $(\%)$} \\
\hline & $\operatorname{VaR}_{S_{2}}(0.99)$ & $T V a R_{S_{2}}(0.99)$ & $\operatorname{Va} R_{S_{2}}(0.99)$ & $T V a R_{S_{2}}(0.99)$ & $\operatorname{Va} R_{S_{2}}(0.99)$ & $T V a R_{S_{2}}(0.99)$ \\
\hline-1.91 & 12.24 & 13.92 & 12.26 & 13.91 & -0.16 & 0.10 \\
\hline-0.87 & 12.35 & 14.04 & 12.38 & 14.03 & -0.25 & 0.06 \\
\hline 0 & 12.44 & 14.13 & 12.48 & 14.13 & -0.31 & 0.03 \\
\hline 0.87 & 12.53 & 14.22 & 12.57 & 14.22 & -0.29 & 0.01 \\
\hline 1.87 & 12.62 & 14.31 & 12.66 & 14.32 & -0.33 & -0.02 \\
\hline 2.87 & 12.71 & 14.41 & 12.74 & 14.41 & -0.24 & -0.05 \\
\hline 3.87 & 12.80 & 14.49 & 12.82 & 14.50 & -0.14 & -0.08 \\
\hline 4.87 & 12.88 & 14.57 & 12.90 & 14.59 & -0.14 & -0.10 \\
\hline
\end{tabular}

their risks. In the literature, many capital allocation techniques have been developed, see Cummins (2000), Dhaene et al. (2012), McNeil et al. (2005), and Tasche (2004) and references therein. In practice, the TVaR and the covariance allocation principle are commonly used, since they take into account the dependence structure between risks. More precisely, if $S_{n}=\sum_{i=1}^{n} X_{i}$ is the aggregate risk where $X_{i}$ is a continuous rv with finite mean that represents the individual risk, the amount of capital $T_{i}$ allocated to each risk $X_{i}$, for $i=1, \ldots, n$, is defined as ( for a tolerance level $p \in(0,1)$, denote $T_{i}=T \operatorname{Va} R_{p}\left(X_{i}, S_{n}\right)$ under the TVaR allocation principle, $T_{i}=K_{p}\left(X_{i}, S_{n}\right)$ under the covariance allocation principle)

$$
\begin{gathered}
\operatorname{TVa}_{p}\left(X_{i}, S_{n}\right)=\frac{\mathbb{E}\left(X_{i} \mathbb{1}_{\left\{S_{n}>\operatorname{VaR}_{S_{n}}(p)\right\}}\right)}{1-p}, \\
K_{p}\left(X_{i}, S_{n}\right)=\mathbb{E}\left(X_{i}\right)+\frac{\operatorname{Cov}\left(X_{i}, S_{n}\right)}{\operatorname{Var}\left(S_{n}\right)}\left(T \operatorname{Va} R_{S_{n}}(p)-\mathbb{E}\left(S_{n}\right)\right),
\end{gathered}
$$

where we assume that $S_{n}$ has finite and positive variance. We have

$$
\sum_{i=1}^{n} T_{i}=\sum_{i=1}^{n} T \operatorname{Va} R_{p}\left(X_{i}, S_{n}\right)=\sum_{i=1}^{n} K_{p}\left(X_{i}, S_{n}\right)=T \operatorname{Va} R_{S_{n}}(p),
$$

which means that for both allocation principle, based on TVaR as a risk measure, the capital required for the entire portfolio is equal to the sum of the allocated capital of each risk within the portfolio. Given some vector $V=$ $\left(v_{1}, v_{2}, \ldots\right)$ with non-negative components such that $\sum_{j=1}^{\infty} j v_{j}<\infty$ we define the new vector $G(L)=\left(g_{1}, g_{2}, \ldots\right)$ where

$$
g_{k}= \begin{cases}0 & \text { for } k=1 \\ \frac{(k-1) v_{k-1}}{\sum_{j=1}^{\infty} j v_{j}} & \text { for } k>1 .\end{cases}
$$


For notational simplicity we shall also write in the following $\overline{\beta_{i}}$ instead of $\beta_{i} /\left(\beta_{i}+1\right)$. Furthermore hereafter the df of the pdf given in (2.11) will be denoted by $W_{k}(\cdot, \beta)$ with survival function $\bar{W}_{k}(\cdot, \beta)$.

We derive next an explicit form of $T \operatorname{Va} R_{p}\left(X_{i}, S_{2}\right)$ and $K_{p}\left(X_{i}, S_{2}\right), i=1,2$, in the case of SmE type risks.

Proposition 4.1. Let $\left(X_{1}, X_{2}\right) \sim S M E_{2}\left(\boldsymbol{\beta}, Q_{1}, Q_{2}\right)$ with $\beta_{1} \leqslant \beta_{2}$, further let $\Theta_{i}$ and $\Psi_{i}$ be defined as in Proposition 3.1. If for $i=1,2$ both $\mu_{i}:=\frac{1}{\beta_{i}} \sum_{k=1}^{\infty} k q_{i, k}$ and $\tilde{\mu}_{i}:=\frac{1}{\beta_{i}+1} \sum_{k=1}^{\infty} k \theta_{i, k}$ are finite, then for any $p \in(0,1)$ the amount of capital allocated to each risk $X_{i}, i=1,2$, under the TVaR principle is

$$
\operatorname{TVa}_{p}\left(X_{i}, S_{2}\right)=\frac{1}{1-p} \sum_{k=1}^{\infty} z_{i k} \bar{W}_{k}\left(\operatorname{VaR}_{S_{2}}(p), \beta_{2}+1\right),
$$

where $\gamma=\alpha_{12} \mathcal{L}_{1} \mathcal{L}_{2}$,

$$
\begin{aligned}
z_{i, k}= & (1+\gamma) \mu_{i} \pi_{k}\left\{\Psi_{i}\left(G_{i}\left(Q_{i}\right)\right), \Psi_{j}\left(Q_{j}\right)\right\}+\gamma \tilde{\mu}_{i} \pi_{k}\left\{\Psi_{i}\left(G_{i}\left(\Theta_{i}\right)\right), \Psi_{j}\left(\Theta_{j}\right)\right\} \\
& -\gamma \tilde{\mu}_{i} \pi_{k}\left\{\Psi_{i}\left(G_{i}\left(\Theta_{i}\right)\right), \Psi_{j}\left(Q_{j}\right)\right\}-\gamma \mu_{i} \pi_{k}\left\{\Psi_{i}\left(G_{i}\left(Q_{i}\right)\right), \Psi_{j}\left(\Theta_{j}\right)\right\}, \quad i \neq j,
\end{aligned}
$$

and the contribution of each risk $X_{i}, i=1,2$ to the economic capital of the entire portfolio, under the covariance principle, is given by

$$
K_{p}\left(X_{i}, S_{2}\right)=\sum_{k=1}^{\infty} \frac{L_{i, k}}{\beta_{2}+1},
$$

where

$$
L_{i, k}=k \psi_{i, k}+\varepsilon_{i, j}\left(\frac{P_{k}^{*}\left(\left(\beta_{2}+1\right) V a R_{S_{2}}(p)\right)^{k}}{\varphi k !}+\left(\beta_{2}+1\right) V a R_{S_{2}}(p)-k p_{k}\right), i \neq j,
$$

with

$$
\begin{aligned}
\varepsilon_{i, j}= & \frac{\sum_{m=1}^{\infty}\left(m^{2}+m\right) \psi_{i m}-\left(\sum_{m=1}^{\infty} m \psi_{i m}\right)^{2}}{\sum_{m=1}^{\infty}\left(m^{2}+m\right) p_{m}-\left(\sum_{m=1}^{\infty} m p_{m}\right)^{2}} \\
& +\left(\frac{\alpha_{12}\left(\beta_{2}+1\right)^{2}}{\sum_{m=1}^{\infty}\left(m^{2}+m\right) p_{m}-\left(\sum_{m=1}^{\infty} m p_{m}\right)^{2}}\right) \\
& \times\left(\frac{1}{\beta_{i}+1} \sum_{m=1}^{\infty} m q_{i, m}{\overline{\beta_{i}}}^{m}-\frac{1}{\beta_{i}} \sum_{m=1}^{\infty} q_{i, m}{\overline{\beta_{i}}}^{m} \sum_{m=1}^{\infty} m q_{i, m}\right) \\
& \times\left(\frac{1}{\beta_{j}+1} \sum_{m=1}^{\infty} m q_{j, m}{\overline{\beta_{j}}}^{m}-\frac{1}{\beta_{j}} \sum_{m=1}^{\infty} q_{j, m}{\overline{\beta_{j}}}^{m} \sum_{m=1}^{\infty} m q_{j, m}\right),
\end{aligned}
$$


TABLE 4.1

ANALYTICAL FOR MULA: DEPENDENCE LEVEL, TVAR AND ALLOCATED CAPITAL TO EACH RISK $X_{i}, i=1,2$, UNDER THE TVAR AND THE COVARIANCE CAPITAL ALLOCATION PRINCIPLE.

\begin{tabular}{cccccc}
\hline \hline$\alpha_{12}$ & $T V a R_{S_{2}}(0.99)$ & $T V a R_{0.99}\left(X_{1}, S_{2}\right)$ & $T V a R_{0.99}\left(X_{2}, S_{2}\right)$ & $K_{0.99}\left(X_{1}, S_{2}\right)$ & $K_{0.99}\left(X_{2}, S_{2}\right)$ \\
\hline-1.91 & 13.92 & 7.70 & 6.22 & 7.69 & 6.23 \\
-0.87 & 14.04 & 7.74 & 6.30 & 7.73 & 6.31 \\
0 & 14.13 & 7.77 & 6.36 & 7.75 & 6.38 \\
0.87 & 14.22 & 7.80 & 6.42 & 7.78 & 6.44 \\
1.87 & 14.31 & 7.84 & 6.47 & 7.81 & 6.50 \\
2.87 & 14.41 & 7.87 & 6.54 & 7.84 & 6.57 \\
3.87 & 14.49 & 7.90 & 6.59 & 7.87 & 6.62 \\
4.87 & 14.57 & 7.93 & 6.64 & 7.89 & 6.68 \\
\hline \hline
\end{tabular}

$$
\begin{aligned}
\varphi & =\sum_{j=1}^{\infty} \frac{P_{j}\left(\left(\beta_{2}+1\right) V a R_{S_{2}}(p)\right)^{j-1}}{(j-1) !}, \quad P_{k}^{*}=\sum_{j=k}^{\infty} P_{j}, \\
P_{j} & =\frac{\sum_{k=j}^{\infty} p_{k}}{\sum_{k=1}^{\infty} k p_{k}} \quad \text { and } p_{k} \text { is given in (3.1). }
\end{aligned}
$$

Example 4.2. In this example, we consider the same marginals and dependence parameters as in Example 3.2. For different level of the dependence between $X_{1}$ and $X_{2}$, which is described by $\alpha_{12}$, TVaRs have been calculated on the aggregated risk $S_{2}=X_{1}+X_{2}$ at a tolerance level $p=99 \%$. Furthermore, the allocated capital to each risk $X_{i}, i=1,2$, under the TVaR and the covariance capital allocation principle are also evaluated. Table 4.1 demonstrates that risk measures on the aggregated risk are sensitive to the level of dependence between individual risks. Actually, due to the relationship between dependence level and the diversification effect, the more $X_{1}$ and $X_{2}$ are dependent, the more the portfolio is risky, hence more capital is needed to cover the risks. In this respect, more capital is allocated to risk $X_{1}$ compared to the amount allocated to risk $X_{2}$ under the TVaR and the covariance principle.

\section{AuXiLIARy Results}

One of the main features of the mixed Erlang distribution is that its pdf can be used to derive some results in an analytical way. In this respect, this section presents some useful properties of the mixed Erlang distribution.

Lemma 5.1. If $X$ is a random variable from the mixed Erlang distribution with $p d f$ $g(x, \beta, Q)$, then $g^{\theta}(x, \beta+1, \Theta)=\frac{e^{-x} g(x, \beta, Q)}{\mathcal{L}}$, with $\mathcal{L}=\mathbb{E}\left(e^{-X}\right)$, is again a $p d f$ of the mixed Erlang distribution with mixing probabilities $\Theta=\left(\theta_{1}, \theta_{2}, \ldots\right)$ and 
scale parameter $\beta+1$ and we have

$$
g^{\theta}(x, \beta+1, \Theta)=\sum_{k=1}^{\infty} \theta_{k} w_{k}(x, \beta+1),
$$

where $\theta_{k}=\frac{q_{k} \bar{\beta}^{k}}{\sum_{j=1}^{\infty} q_{j} \bar{\beta}^{j}}$ with $\bar{\beta}=\frac{\beta}{\beta+1}$.

The result presented in the next two lemmas can be found in Section 2.2 of Willmot and Woo (2007), and Section 7.2 of Lee and Lin (2010), respectively.

Lemma 5.2. If $X \sim \operatorname{ME}\left(\beta_{1}, Q\right)$, then for any positive constant $\beta_{2} \geq \beta_{1}$ we have

$$
X \sim \operatorname{ME}\left(\beta_{2}, \Psi(Q)\right),
$$

where the mixing probabilities $\Psi(Q)=\left(\psi_{1}, \psi_{2}, \ldots\right)$ and its individual components are given by

$$
\psi_{k}=\sum_{i=1}^{k} q_{i}\left(\begin{array}{c}
k-1 \\
i-1
\end{array}\right)\left(\frac{\beta_{1}}{\beta_{2}}\right)^{i}\left(1-\frac{\beta_{1}}{\beta_{2}}\right)^{k-i}, \quad k \geq 1 .
$$

Lemma 5.3. Let $X_{1}, X_{2}$ be two independent random variables. If $X_{i} \sim$ $\operatorname{ME}\left(\beta_{i}, Q_{i}\right), i=1,2$, then $S_{2}=X_{1}+X_{2} \sim \operatorname{ME}\left(\beta, \prod\left\{Q_{1}, Q_{2}\right\}\right)$, provided that $\beta_{1}=\beta_{2}=\beta$ with

$$
\pi_{l}\left\{Q_{1}, Q_{2}\right\}=\left\{\begin{array}{lll}
0 & \text { for } & l=1 \\
\sum_{j=1}^{l-1} q_{1, j} q_{2, l-j} & \text { for } & l>1 .
\end{array}\right.
$$

Remarks 5.4. According to Cossette et al. (2012) (Remark 2.1), the results in Lemma 5.3 can be extended to $S_{n}=\sum_{i=1}^{n} X_{i}$, as long as $X_{i}, \ldots, X_{n}$ are independent, $X_{i} \sim \operatorname{ME}\left(\beta_{i}, Q_{i}\right)$ and $\beta_{i}=\beta$ for $i=1, \ldots, n$. Specifically, $S_{n} \sim \operatorname{ME}\left(\beta, \prod\left\{Q_{1}, \ldots, Q_{n}\right\}\right)$ where the individual mixing probabilities can be evaluated iteratively as follows

$\pi_{l}\left\{Q_{1}, \ldots, Q_{n+1}\right\}= \begin{cases}0 & \text { for } l=1, \ldots, n \\ \sum_{j=n}^{l-1} \pi_{j}\left\{Q_{1}, \ldots, Q_{n}\right\} q_{n+1, l-j} & \text { for } l=n+1, n+2, \ldots\end{cases}$

\section{Multivariate SmE Risks}

In this section, we assume that the joint distribution of the random vector $\left(X_{1}, \ldots, X_{n}\right)$ will be referred to as a multivariate $\operatorname{SmE}$ distribution and we shall abbreviate this as $\left(X_{1}, \ldots, X_{n}\right) \sim \operatorname{SME}_{n}\left(\boldsymbol{\beta}, Q_{1}, \ldots, Q_{n}\right)$ where 
$\boldsymbol{\beta}=\left(\beta_{1}, \ldots, \beta_{n}\right)$ with $X_{i} \sim \operatorname{ME}\left(\beta_{i}, Q_{i}\right), i=1, \ldots, n$. Furthermore, we shall set

$$
\tilde{f}_{i}\left(x_{i}\right):=e^{-x_{i}} f_{i}\left(x_{i}\right) \text {. }
$$

\subsection{Distribution of $S_{n}$}

By decomposing the joint pdf of $\left(X_{1}, \ldots, X_{n}\right)$ in (2.10) and using some rules of integration, we show in the next proposition that the distribution of $S_{n}=$ $\sum_{i=1}^{n} X_{i}$ belongs to the class of Erlang mixtures.

Proposition 6.1. If $\left(X_{1}, \ldots, X_{n}\right) \sim S M E_{n}\left(\boldsymbol{\beta}, Q_{1}, \ldots, Q_{n}\right)$ with $\beta_{i} \leqslant \beta_{n}$, for $i=$ $1, \ldots, n-1$, then $S_{n} \sim M E\left(\beta_{n}+1, P\right)$. The components of $\mathcal{D}=\left(p_{1}, p_{2}, \ldots\right)$ are given by

$$
\begin{aligned}
p_{k}= & \left(1+\sum_{j_{1}} \sum_{j_{2}} \alpha_{j_{1}, j_{2}} \mathcal{L}_{j_{1}} \mathcal{L}_{j_{2}}-\sum_{j_{1}} \sum_{j_{2}} \sum_{j_{3}} \alpha_{j_{1}, j_{2}, j_{3}} \mathcal{L}_{j_{1}} \mathcal{L}_{j_{2}} \mathcal{L}_{j_{3}}\right. \\
& \left.+\cdots+(-1)^{n} \alpha_{1,2, \ldots, n} \prod_{i=1}^{n} \mathcal{L}_{i}\right) \bar{\pi}^{(k)}+\sum_{j_{1}}\left(-\sum_{j_{2}} \alpha_{j_{1}, j_{2}} \mathcal{L}_{j_{2}}\right. \\
& \left.+\sum_{j_{2}} \sum_{j_{3}} \alpha_{j_{1}, j_{2}, j_{3}} \mathcal{L}_{j_{2}} \mathcal{L}_{j_{3}}+\cdots+(-1)^{n+1} \alpha_{1,2, \ldots, n} \prod_{i \in C \backslash\left\{j_{1}\right\}} \mathcal{L}_{i}\right) \bar{\pi}_{j 1}^{(k)} \\
& +\sum_{j_{1}} \sum_{j_{2}}\left(\alpha_{j_{1}, j_{2}}-\sum_{j_{3}} \alpha_{j_{1}, j_{2}, j_{3}} \mathcal{L}_{j_{3}}+\sum_{j_{3}} \sum_{j_{4}} \alpha_{j_{1}, j_{2}, j_{3}, j_{4}} \mathcal{L}_{j_{3}} \mathcal{L}_{j_{4}}\right. \\
& \left.+\cdots+(-1)^{n} \alpha_{1,2, \ldots, n} \prod_{i \in C \backslash\left\{j_{1}, j_{2}\right\}} \mathcal{L}_{i}\right) \bar{\pi}_{j 1, j_{2}}^{(k)} \\
& +\sum_{j_{1}} \sum_{j_{2}} \sum_{j_{3}}\left(\alpha_{j_{1}, j_{2}, j_{3}}-\sum_{j_{4}} \alpha_{j_{1}, j_{2}, j_{3}, j_{4}} \mathcal{L}_{j_{4}}+\sum_{j_{4}} \sum_{j_{5}} \alpha_{j_{1}, j_{2}, j_{3}, j_{4}, j_{5}} \mathcal{L}_{j_{4}} \mathcal{L}_{j_{5}}\right. \\
& \left.+\cdots+(-1)^{n+1} \alpha_{1,2, \ldots, n} \prod_{i \in C \backslash\left\{j_{1}, j_{2}\right\}} \mathcal{L}_{i}\right) \bar{\pi}_{j 1, j_{2}, j_{3}}^{(k)} \\
& +\cdots+\sum_{j_{1}} \sum_{j_{2}} \ldots \sum_{j_{n-1}}\left(\alpha_{j_{1}, j_{2}, \ldots, j_{n-1}}-\alpha_{1,2, \ldots, n} \mathcal{L}_{j_{n}}\right) \bar{\pi}_{j 1, \ldots, j_{n-1}}^{(k)}+\alpha_{1,2, \ldots, n} \bar{\pi}_{1, \ldots, n}^{(k)},
\end{aligned}
$$

where

$$
\begin{aligned}
& \bar{\pi}^{(k)}=\pi_{k}\left\{\Psi_{1}\left(Q_{1}\right), \ldots, \Psi_{n}\left(Q_{n}\right)\right\}, \\
& \bar{\pi}_{j_{1}}^{(k)}=\mathcal{L}_{j_{1}} \pi_{k}\left\{\Psi_{j_{1}}\left(\Theta_{j_{1}}\right), \Psi_{j_{2}}\left(Q_{j_{2}}\right), \ldots, \Psi_{n}\left(Q_{n}\right)\right\}, \\
& \bar{\pi}_{j_{1}, j_{2}}^{(k)}=\mathcal{L}_{j_{1}} \mathcal{L}_{j_{2}} \pi_{k}\left\{\Psi_{j_{1}}\left(\Theta_{j_{1}}\right), \Psi_{j_{2}}\left(\Theta_{j_{2}}\right), \Psi_{j_{3}}\left(Q_{j_{3}}\right) \ldots, \Psi_{n}\left(Q_{n}\right)\right\},
\end{aligned}
$$


$\bar{\pi}_{j_{1}, j_{2}, j_{3}}^{(k)}=\mathcal{L}_{j_{1}} \mathcal{L}_{j_{2}} \mathcal{L}_{j_{3}} \pi_{k}\left\{\Psi_{j_{1}}\left(\Theta_{j_{1}}\right), \Psi_{j_{2}}\left(\Theta_{j_{2}}\right), \Psi_{j_{3}}\left(\Theta_{j_{3}}\right) \ldots, \Psi_{n}\left(Q_{n}\right)\right\}$, $\bar{\pi}_{j_{1}, \ldots, j_{n-1}}^{(k)}=\mathcal{L}_{j_{1}} \cdots \mathcal{L}_{j_{n-1}} \pi_{k}\left\{\Psi_{j_{1}}\left(\Theta_{j_{1}}\right), \ldots, \Psi_{j_{n-1}}\left(\Theta_{j_{n-1}}\right), \Psi_{j_{n}}\left(Q_{j_{n}}\right)\right\}$, $\bar{\pi}_{1, \ldots, n}^{(k)}=\mathcal{L}_{1} \cdots \mathcal{L}_{n} \pi_{k}\left\{\Psi_{1}\left(\Theta_{1}\right), \ldots, \Psi_{n}\left(\Theta_{n}\right)\right\}$, with $C=\{1, \ldots, n\}, j_{1} \in C, j_{2} \in C \backslash\left\{j_{1}\right\}, j_{3} \in C \backslash\left\{j_{1}, j_{2}\right\}, \ldots, j_{n} \in$ $C \backslash\left\{j_{1}, \ldots, j_{n-1}\right\}$.

Example 6.2. Let $\left(X_{1}, X_{2}, X_{3}\right) \sim S M E_{3}\left(\boldsymbol{\beta}, Q_{1}, Q_{2}, Q_{3}\right)$ with $\beta_{i} \leqslant \beta_{3}, i=1,2$ then $S_{3} \sim M E\left(\beta_{3}+1, P\right)$ where the components of $\mathcal{X}=\left(p_{1}, p_{2}, \ldots\right)$ are given by (with $C=\{1,2,3\}$ )

$$
\begin{aligned}
p_{k}= & \left(1+\sum_{j_{1}} \sum_{j_{2}} \alpha_{j_{1}, j_{2}} \mathcal{L}_{j_{1}} \mathcal{L}_{j_{2}}-\alpha_{1,2,3} \prod_{i=1}^{3} \mathcal{L}_{i}\right) \bar{\pi}^{(k)} \\
& +\sum_{j_{1}}\left(-\sum_{j_{2}} \alpha_{j_{1}, j_{2}} \mathcal{L}_{j_{2}}+\sum_{j_{2}} \sum_{j_{3}} \alpha_{j_{1}, j_{2}, j_{3}} \mathcal{L}_{j_{2}} \mathcal{L}_{j_{3}}+\alpha_{1,2,3} \prod_{i \in C \backslash\left\{j_{1}\right\}} \mathcal{L}_{i}\right) \bar{\pi}_{j 1}^{(k)} \\
& +\sum_{j_{1}} \sum_{j_{2}}\left(\alpha_{j_{1}, j_{2}}-\alpha_{1,2,3} \mathcal{L}_{j_{3}}\right) \bar{\pi}_{j_{1}, j_{2}}^{(k)}+\alpha_{1,2,3} \bar{\pi}_{1,2,3}^{(k)} \\
= & \left(1+\alpha_{1,2} \mathcal{L}_{1} \mathcal{L}_{2}+\alpha_{1,3} \mathcal{L}_{1} \mathcal{L}_{3}+\alpha_{2,3} \mathcal{L}_{2} \mathcal{L}_{3}-\alpha_{1,2,3} \mathcal{L}_{1} \mathcal{L}_{2} \mathcal{L}_{3}\right) \\
& \times \pi_{k}\left\{\Psi_{1}\left(Q_{1}\right), \Psi_{2}\left(Q_{2}\right), \Psi_{3}\left(Q_{3}\right)\right\} \\
& +\left(-\alpha_{1,2} \mathcal{L}_{2}-\alpha_{1,3} \mathcal{L}_{3}+\alpha_{1,2,3} \mathcal{L}_{2} \mathcal{L}_{3}\right) \mathcal{L}_{1} \pi_{k}\left\{\Psi_{1}\left(\Theta_{1}\right), \Psi_{2}\left(Q_{2}\right), \Psi_{3}\left(Q_{3}\right)\right\} \\
& +\left(-\alpha_{1,2} \mathcal{L}_{1}-\alpha_{2,3} \mathcal{L}_{3}+\alpha_{1,2,3} \mathcal{L}_{1} \mathcal{L}_{3}\right) \mathcal{L}_{2} \pi_{k}\left\{\Psi_{1}\left(Q_{1}\right), \Psi_{2}\left(\Theta_{2}\right), \Psi_{3}\left(Q_{3}\right)\right\} \\
& +\left(-\alpha_{1,3} \mathcal{L}_{1}-\alpha_{2,3} \mathcal{L}_{2}+\alpha_{1,2,3} \mathcal{L}_{1} \mathcal{L}_{2}\right) \mathcal{L}_{3} \pi_{k}\left\{\Psi_{1}\left(Q_{1}\right), \Psi_{2}\left(Q_{2}\right), \Psi_{3}\left(\Theta_{3}\right)\right\} \\
& +\left(\alpha_{1,3}-\alpha_{1,2,3} \mathcal{L}_{2}\right) \mathcal{L}_{1} \mathcal{L}_{3} \pi_{k}\left\{\Psi_{1}\left(\Theta_{1}\right), \Psi_{2}\left(Q_{2}\right), \Psi_{3}\left(\Theta_{3}\right)\right\} \\
& +\left(\alpha_{2,3}-\alpha_{1,2,3} \mathcal{L}_{1}\right) \mathcal{L}_{2} \mathcal{L}_{3} \pi_{k}\left\{\Psi_{1}\left(Q_{1}\right), \Psi_{1}\left(\Theta_{1}\right), \Psi_{3}\left(\Theta_{3}\right)\right\} \\
& +\left(\alpha_{1,2}-\alpha_{1,2,3} \mathcal{L}_{3}\right) \mathcal{L}_{1} \mathcal{L}_{2} \pi_{k}\left\{\Psi_{1}\left(\Theta_{1}\right), \Psi_{2}\left(\Theta_{2}\right), \Psi_{3}\left(Q_{3}\right)\right\} \\
& +\alpha_{1,2,3} \mathcal{L}_{1} \mathcal{L}_{2} \mathcal{L}_{3} \pi_{k}\left\{\Psi_{1}\left(\Theta_{1}\right), \Psi_{2}\left(\Theta_{2}\right), \Psi_{3}\left(\Theta_{3}\right)\right\}
\end{aligned}
$$

\subsection{Capital allocation}

The following propositions provide analytical formulas for the allocated capital to each individual risk $X_{m}, m=1, \ldots, n$, under the TVaR and the covariance rules.

Proposition 6.3. Let $\left(X_{1}, \ldots, X_{n}\right) \sim S M E_{n}\left(\boldsymbol{\beta}, Q_{1}, \ldots, Q_{n}\right)$ with $\beta_{m} \leqslant \beta_{n}$, for $m=1, \ldots, n-1$. Provided that both $\mu_{m}=\frac{1}{\beta_{m}} \sum_{k=1}^{\infty} k q_{m, k}$ and $\tilde{\mu}_{m}=$ $\frac{1}{\beta_{m}+1} \sum_{k=1}^{\infty} k \theta_{m k}, m=1, \ldots, n$ are finite, then for $m=1, \ldots, n$ and $p \in(0,1)$ the amount of capital allocated to each risk $X_{m}$ under the TVaR principle is given 
by $(\operatorname{set} C:=\{1, \ldots, n\})$

$$
T \operatorname{Va} R_{p}\left(X_{m}, S_{n}\right)=\frac{1}{1-p} \sum_{k=1}^{\infty} z_{m, k} \bar{W}_{k}\left(\operatorname{VaR}_{S_{n}}(p), \beta_{n}+1\right),
$$

where

$$
\begin{aligned}
z_{m, k}= & \left(1+\sum_{j_{1}} \sum_{j_{2}} \alpha_{j_{1}, j_{2}} \mathcal{L}_{j_{1}} \mathcal{L}_{j_{2}}-\sum_{j_{1}} \sum_{j_{2}} \sum_{j_{3}} \alpha_{j_{1}, j_{2}, j_{3}} \mathcal{L}_{j_{1}} \mathcal{L}_{j_{2}} \mathcal{L}_{j_{3}}+\cdots\right. \\
& \left.+(-1)^{n} \alpha_{1,2, \ldots, n} \prod_{i=1}^{n} \mathcal{L}_{i}\right) \mu_{m} \tilde{\pi}^{(k)}+\sum_{j_{1} \neq m}\left(-\sum_{j_{2}} \alpha_{j_{1}, j_{2}} \mathcal{L}_{j_{2}}+\sum_{j_{2}} \sum_{j_{3}} \alpha_{j_{1}, j_{2}, j_{3}}\right. \\
& \left.\times \mathcal{L}_{j_{2}} \mathcal{L}_{j_{3}}+\cdots+(-1)^{n+1} \alpha_{1,2, \ldots, n} \prod_{i \in C \backslash\left\{j_{1}\right\}} \mathcal{L}_{i}\right) \mu_{m} \tilde{\pi}_{j_{1}}^{(k)}+\left(-\sum_{j_{2} \neq m} \alpha_{m, j_{2}} \mathcal{L}_{j_{2}}\right. \\
& \left.+\sum_{j_{2} \neq m} \sum_{j_{3} \neq m} \alpha_{m, j_{2}, j_{3}} \mathcal{L}_{j_{2}} \mathcal{L}_{j_{3}}+\cdots+(-1)^{n+1} \alpha_{1,2, \ldots, n} \prod_{i \in C \backslash\{m\}} \mathcal{L}_{i}\right) \tilde{\mu}_{m} \tilde{\pi}_{m}^{(k)} \\
& +\sum_{j_{1} \neq m} \sum_{j_{2}}\left(\alpha_{j_{1}, j_{2}}-\sum_{j_{3}} \alpha_{j_{1}, j_{2}, j_{3}} \mathcal{L}_{j_{3}}+\sum_{j_{3}} \sum_{j_{4}} \alpha_{j_{1}, j_{2}, j_{3}, j_{4}} \mathcal{L}_{j_{3}} \mathcal{L}_{j_{4}}+\cdots\right. \\
& \left.+(-1)^{n} \alpha_{1,2, \ldots, n} \prod_{i \in C \backslash\left\{j_{1}, j_{2}\right\}} \mathcal{L}_{i}\right) \mu_{m} \tilde{\pi}_{j_{1}, j_{2}}^{(k)}+\sum_{j_{2} \neq m}\left(\alpha_{m, j_{2}}-\sum_{j_{3} \neq m} \alpha_{m, j_{2}, j_{3}} \mathcal{L}_{j_{3}}\right. \\
& \left.+\sum_{j_{3} \neq m} \sum_{j_{4} \neq m} \alpha_{m, j_{2}, j_{3}, j_{4}} \mathcal{L}_{j_{3}} \mathcal{L}_{j_{4}}+\ldots+(-1)^{n} \alpha_{1,2, \ldots, n} \prod_{i \in C \backslash\left\{m, j_{2}\right\}} \mathcal{L}_{i}\right) \tilde{\mu}_{m} \tilde{\pi}_{m, j_{2}}^{(k)} \\
& +\ldots+\sum_{j_{1} \neq m} \sum_{j_{2}} \ldots \sum_{j_{n-1}}\left(\alpha_{j_{1}, j_{2}, \ldots, j_{n-1}}-\alpha_{1,2, \ldots, n} \mathcal{L}_{m}\right) \mu_{m} \tilde{\pi}_{j_{1}, \ldots, j_{n-1}}^{(k)} \\
& +\sum_{j_{2} \neq m} \ldots \sum_{j_{n-1} \neq m}\left(\alpha_{m, j_{2}, \ldots, j_{n-1}}-\alpha_{1,2, \ldots, n} \mathcal{L}_{j_{n} \neq m}\right) \tilde{\mu}_{m} \tilde{\pi}_{m, j_{2}, \ldots, j_{n-1}}^{(k)}+\alpha_{1,2, \ldots, n} \tilde{\pi}_{1, \ldots, n}^{(k)},
\end{aligned}
$$

where

$$
\begin{aligned}
& \tilde{\pi}^{(k)}=\pi_{k}\left\{\Psi_{m}\left(G_{m}\left(Q_{m}\right), \Psi_{1}\left(Q_{1}\right), \ldots, \Psi_{n}\left(Q_{n}\right)\right\},\right. \\
& \tilde{\pi}_{j_{1}}^{(k)}=\mathcal{L}_{j_{1}} \pi_{k}\left\{\Psi_{m}\left(G_{m}\left(Q_{m}\right), \Psi_{j_{1}}\left(\Theta_{j_{1}}\right), \ldots, \Psi_{n}\left(Q_{n}\right)\right\},\right. \\
& \tilde{\pi}_{m}^{(k)}=\mathcal{L}_{m} \pi_{k}\left\{\Psi_{m}\left(G_{m}\left(\Theta_{m}\right), \ldots, \Psi_{n}\left(Q_{n}\right)\right\},\right. \\
& \tilde{\pi}_{j_{1}, j_{2}}^{(k)}=\mathcal{L}_{j_{1}} \mathcal{L}_{j_{2}} \pi_{k}\left\{\Psi_{m}\left(G_{m}\left(Q_{m}\right), \Psi_{j_{1}}\left(\Theta_{j_{1}}\right), \Psi_{j_{2}}\left(\Theta_{j_{2}}\right), \ldots, \Psi_{n}\left(Q_{n}\right)\right\},\right. \\
& \tilde{\pi}_{m, j_{2}}^{(k)}=\mathcal{L}_{m} \mathcal{L}_{j_{2}} \pi_{k}\left\{\Psi_{m}\left(G_{m}\left(\Theta_{m}\right), \Psi_{j_{2}}\left(\Theta_{j_{2}}\right), \ldots, \Psi_{n}\left(Q_{n}\right)\right\},\right. \\
& \tilde{\pi}_{j_{1}, \ldots, j_{n-1}}^{(k)}=\mathcal{L}_{j_{1}} \cdots \mathcal{L}_{j_{n-1}} \pi_{k}\left\{\Psi_{m}\left(G_{m}\left(Q_{m}\right), \Psi_{j_{1}}\left(\Theta_{j_{1}}\right) \ldots, \Psi_{n}\left(\Theta_{j_{n-1}}\right)\right\},\right.
\end{aligned}
$$


$\tilde{\pi}_{m, j_{2}, \ldots, j_{n-1}}^{(k)}=\mathcal{L}_{m} \mathcal{L}_{j_{2}} \cdots \mathcal{L}_{j_{n-1}} \pi_{k}\left\{\Psi_{m}\left(G_{m}\left(\Theta_{m}\right), \Psi_{j_{2}}\left(\Theta_{j_{2}}\right) \ldots, \Psi_{j_{n-1}}\left(\Theta_{j_{n-1}}\right), \Psi_{j_{n}}\left(Q_{j_{n}}\right)\right\}\right.$ $\tilde{\pi}_{1, \ldots, n}^{(k)}=\mathcal{L}_{m} \mathcal{L}_{1} \cdots \mathcal{L}_{n} \pi_{k}\left\{\Psi_{m}\left(G_{m}\left(\Theta_{m}\right), \Psi_{j_{2}}\left(\Theta_{j_{2}}\right), \ldots, \Psi_{n}\left(\Theta_{j_{n}}\right\}\right.\right.$.

Proposition 6.4. Let $\beta_{m} \leqslant \beta_{n}, m \leq n-1$, and consider $\left(X_{1}, \ldots, X_{n}\right) \sim$ $S M E_{n}\left(\boldsymbol{\beta}, Q_{1}, \ldots, Q_{n}\right)$. If $S_{n}$ has a finite and positive variance, then for any index $m \leq n$ and $p \in(0,1)$ we have

$$
K_{p}\left(X_{m}, S_{n}\right)=\sum_{k=1}^{\infty} \frac{L_{m, k}}{\beta_{n}+1},
$$

where $K_{p}$ is defined in (4.2),

$$
L_{m, k}=k \psi_{m, k}+\varepsilon_{m, j}\left(\frac{P_{k}^{*}\left(\left(\beta_{n}+1\right) V a R_{S_{n}}(p)\right)^{k}}{\varphi k !}+\left(\beta_{n}+1\right) V a R_{S_{n}}(p)-k p_{k}\right), m \neq j,
$$

with

$$
\begin{gathered}
\varepsilon_{m, j}=\frac{\sum_{s=1}^{\infty}\left(s^{2}+s\right) \psi_{m, s}-\left(\sum_{s=1}^{\infty} s \psi_{m, s}^{2}\right)}{\sum_{s=1}^{\infty}\left(s^{2}+s\right) p_{s}-\left(\sum_{s=1}^{\infty} s p_{s}\right)^{2}}+\sum_{j=1}^{n}\left(\frac{\alpha_{m j}\left(\beta_{n}+1\right)^{2}}{\sum_{s=1}^{\infty}\left(s^{2}+s\right) p_{s}-\left(\sum_{s=1}^{\infty} s p_{s}\right)^{2}}\right) \\
\times\left(\frac{1}{\beta_{m}+1} \sum_{s=1}^{\infty} s q_{m, s} \bar{\beta}_{m}^{s}-\sum_{s=1}^{\infty} q_{m, s} \bar{\beta}_{m}^{s} \frac{1}{\beta_{m}} \sum_{s=1}^{\infty} s q_{m, s}\right) \\
\times\left(\frac{1}{\beta_{j}+1} \sum_{s=1}^{\infty} s q_{j, s} \bar{\beta}_{j}^{s}-\sum_{s=1}^{\infty} q_{j, s} \bar{\beta}_{j}^{s} \frac{1}{\beta_{j}} \sum_{s=1}^{\infty} s q_{j, s}\right), \\
\varphi=\sum_{s=1}^{\infty} \frac{P_{s}\left(\left(\beta_{n}+1\right) V a R_{S_{n}}(p)\right)^{s-1}}{(s-1) !}, \quad P_{k}^{*}=\sum_{s=k}^{\infty} P_{s}, \\
P_{s}=\frac{\sum_{k=s}^{\infty} p_{s}}{\sum_{s=1}^{\infty} s p_{s}} \quad \text { and } p_{s} \text { is given in (6.1). }
\end{gathered}
$$

Proof. The proof is similar to the bivariate case and is therefore omitted.

\subsection{Trivariate $\mathrm{SmE}$ risks: numerical illustrations}

Let $\left(X_{1}, X_{2}, X_{3}\right)$ have a trivariate SmE risk, with $\alpha_{12}=2.03, \alpha_{13}=3.62, \alpha_{23}=$ -1.54 and $\alpha_{123}=-1.03$ the dependence parameters. The parameters have been chosen so that the condition in (2.9) is fullfilled. Assume $\boldsymbol{\beta}=(0.75,0.9,0.95)$, $Q_{1}=(0.2,0.6,0.2), Q_{2}=(0.4,0.3,0.1,0.2)$ and $Q_{3}=(0.6,0.1,0.2,0.1)$. In view of (2.10) the joint pdf of $\left(X_{1}, X_{2}, X_{3}\right)$ are given by

$$
\begin{aligned}
h(\boldsymbol{x})= & \prod_{i=1}^{3} f_{i}\left(x_{i}\right)\left(2.03\left(e^{-x_{1}}-0.21\right)\left(e^{-x_{2}}-0.28\right)+3.62\left(e^{-x_{1}}-0.21\right)\left(e^{-x_{3}}-0.34\right)\right. \\
& \left.-1.54\left(e^{-x_{2}}-0.28\right)\left(e^{-x_{3}}-0.34\right)-1.03\left(e^{-x_{1}}-0.21\right)\left(e^{-x_{2}}-0.28\right)\left(e^{-x_{3}}-0.34\right)\right) .
\end{aligned}
$$


TABLE 6.1

Mixing PROBABILITIES OF THE DISTRIBUTION OF $S_{3}=X_{1}+X_{2}+X_{3}$, WITH SCALE PARAMETER $\beta_{S_{3}}=1.95$.

\begin{tabular}{cccccccccccc}
\hline \hline$k$ & $p_{k}$ & $k$ & $p_{k}$ & $k$ & $p_{k}$ & $k$ & $p_{k}$ & $k$ & $p_{k}$ & $k$ & $p_{k}$ \\
\hline 1 & 0.0000 & 11 & 0.0670 & 21 & 0.0256 & 31 & 0.0022 & 41 & $8.729 \mathrm{E}-05$ & 51 & $2.150 \mathrm{E}-06$ \\
2 & 0.0000 & 12 & 0.0676 & 22 & 0.0211 & 32 & 0.0017 & 42 & $5.751 \mathrm{E}-05$ & 52 & $1.458 \mathrm{E}-06$ \\
3 & 0.0121 & 13 & 0.0662 & 23 & 0.0172 & 33 & 0.0012 & 43 & $4.289 \mathrm{E}-05$ & 53 & $9.857 \mathrm{E}-07$ \\
4 & 0.0295 & 14 & 0.0631 & 24 & 0.0138 & 34 & 0.0009 & 44 & $2.988 \mathrm{E}-05$ & 54 & $6.648 \mathrm{E}-07$ \\
5 & 0.0366 & 15 & 0.0588 & 25 & 0.0109 & 35 & 0.0006 & 45 & $2.019 \mathrm{E}-05$ & 55 & $4.472 \mathrm{E}-07$ \\
6 & 0.0409 & 16 & 0.0536 & 26 & 0.0086 & 36 & 0.0005 & 46 & $9.869 \mathrm{E}-06$ & 56 & $3.001 \mathrm{E}-07$ \\
7 & 0.0466 & 17 & 0.0478 & 27 & 0.0067 & 37 & 0.0003 & 47 & $9.612 \mathrm{E}-06$ & 57 & $2.010 \mathrm{E}-07$ \\
8 & 0.0533 & 18 & 0.0419 & 28 & 0.0051 & 38 & 0.0002 & 48 & $4.635 \mathrm{E}-06$ & 58 & $1.343 \mathrm{E}-07$ \\
9 & 0.0596 & 19 & 0.0361 & 29 & 0.0039 & 39 & 0.0002 & 49 & $4.513 \mathrm{E}-06$ & 59 & $8.950 \mathrm{E}-08$ \\
10 & 0.0643 & 20 & 0.0307 & 30 & 0.0030 & 40 & 0.0001 & 50 & $3.161 \mathrm{E}-06$ & 60 & $5.795 \mathrm{E}-08$ \\
\hline \hline
\end{tabular}

TABLE 6.2

EXACT VALUES: TVAR OF $S_{3}=X_{1}+X_{2}+X_{3}$ AND ALLOCATED CAPITAL TO EACH RISK $X_{i}, i=1,2,3$, UNDER THE TVAR AND THE COVARIANCE CAPITAL ALLOCATION PRINCIPLE.

\begin{tabular}{lccccccc}
\hline \hline & $\begin{array}{c}T V a R_{S_{3}} \\
p\end{array}$ & $\begin{array}{c}T V a R_{p} \\
\left(X_{1}, S_{3}\right)\end{array}$ & $\begin{array}{c}T V a R_{p} \\
\left(X_{2}, S_{3}\right)\end{array}$ & $\begin{array}{c}T V a R_{p} \\
\left(X_{3}, S_{3}\right)\end{array}$ & $\begin{array}{c}K_{p} \\
\left(X_{1}, S_{3}\right)\end{array}$ & $\begin{array}{c}K_{p} \\
\left(X_{2}, S_{3}\right)\end{array}$ & $\begin{array}{c}K_{p} \\
\left(X_{1}, S_{3}\right)\end{array}$ \\
\hline $90.0 \%$ & 14.16 & 5.53 & 4.73 & 3.90 & 5.56 & 4.70 & 3.90 \\
$92.5 \%$ & 14.84 & 5.79 & 4.96 & 4.09 & 5.84 & 4.93 & 4.07 \\
$95.0 \%$ & 15.77 & 6.13 & 5.29 & 4.35 & 6.20 & 5.23 & 4.34 \\
$97.5 \%$ & 17.29 & 6.70 & 5.82 & 4.77 & 6.82 & 5.72 & 4.75 \\
$99.0 \%$ & 19.20 & 7.45 & 6.47 & 5.28 & 7.58 & 6.35 & 5.27 \\
$99.5 \%$ & 20.58 & 8.01 & 6.94 & 5.63 & 8.13 & 6.80 & 5.65 \\
\hline \hline
\end{tabular}

In light of Proposition 6.1, $S_{3}=X_{1}+X_{2}+X_{3}$ follows the mixed Erlang distribution with scale parameter $\beta_{S_{3}}=1.95$ and mixing probabilities $P=\left(p_{1}, p_{2}, \ldots\right)$, the first 60 values of $P$ are given in Table 6.1. For different tolerance level $p$, Table 6.2 shows the TVaR of $S_{3}=X_{1}+X_{2}+X_{3}$ and the allocated capital to each risk under the covariance and the TVaR capital allocation rules.

\section{PROOFS}

Proof of Proposition 3.1 The pdf $f$ of $S_{2}$ is given in terms of the joint pdf of $\left(X_{1}, X_{2}\right)$ as follows

$$
f_{S_{2}}(s)=\int_{0}^{s} h(y, s-y) d y \text {. }
$$


Taking (2.1) into account the pdf of $S_{2}$ becomes

$$
\begin{aligned}
f_{S_{2}}(s)= & \left(1+\alpha_{12} \mathcal{L}_{1} \mathcal{L}_{2}\right) \int_{0}^{s} f_{1}(y) f_{2}(s-y) d y+\alpha_{12} \int_{0}^{s} e^{-y} f_{1}(y) e^{-(s-y)} f_{2}(s-y) d y \\
& -\alpha_{12} \mathcal{L}_{2} \int_{0}^{s} e^{-y} f_{1}(y) f_{2}(s-y) d y-\alpha_{12} \mathcal{L}_{1} \int_{0}^{s} e^{-(s-y)} f_{2}(s-y) f_{1}(y) d y .
\end{aligned}
$$

Let $A(s), B(s), C(s), D(s)$ be the four terms of the expression of $f_{S_{2}}(s)$ respectively. According to Lemma 5.2,

$$
A(s)=\left(1+\alpha_{12} \mathcal{L}_{1} \mathcal{L}_{2}\right) \int_{0}^{s} f_{1}^{\psi}\left(s, \beta_{2}+1, \Psi_{1}\left(Q_{1}\right)\right) f_{2}^{\psi}\left(s-y, \beta_{2}+1, \Psi_{2}\left(Q_{2}\right)\right) d y
$$

and from Lemma 5.3, $A(s)$ can be expressed as a pdf of the mixed Erlang distribution as follows

$$
A(s)=\left(1+\alpha_{12} \mathcal{L}_{1} \mathcal{L}_{2}\right) \sum_{k=1}^{\infty} \pi_{k}\left(\Psi_{1}\left(Q_{1}\right), \Psi_{2}\left(Q_{2}\right)\right) w_{k}\left(s, \beta_{2}+1\right) .
$$

In view of Lemma 5.1 and Lemma 5.2, the expression of $B(s)$ becomes

$$
\begin{aligned}
B(s) & =\alpha_{12} \int_{0}^{s} \mathcal{L}_{1} f_{1}^{\theta}\left(s, \beta_{1}+1, \Theta_{1}\right) \mathcal{L}_{2} f_{2}^{\theta}\left(s-y, \beta_{2}+1, \Theta_{2}\right) d y \\
& =\alpha_{12} \mathcal{L}_{1} \mathcal{L}_{2} \int_{0}^{s} f_{1}^{\psi}\left(s, \beta_{2}+1, \Psi_{1}\left(\Theta_{1}\right)\right) f_{2}^{\psi}\left(s-y, \beta_{2}+1, \Psi_{2}\left(\Theta_{2}\right)\right) d y .
\end{aligned}
$$

From Lemma 5.3 one can write $B(s)$ as

$$
B(s)=\alpha_{12} \mathcal{L}_{1} \mathcal{L}_{2} \sum_{k=1}^{\infty} \pi_{k}\left(\Psi_{1}\left(\Theta_{1}\right), \Psi_{2}\left(\Theta_{2}\right)\right) w_{k}\left(s, \beta_{2}+1\right),
$$

which is again a pdf of some mixed Erlang distribution. Similarly to $B(s)$, using Lemma 5.1, 5.2 and Lemma 5.3 one can express $C(s)$ and $D(s)$ as pdfs of mixed Erlang distribution as follows

$$
\begin{gathered}
C(s)=\alpha_{12} \mathcal{L}_{1} \mathcal{L}_{2} \sum_{k=1}^{\infty} \pi_{k}\left(\Psi_{1}\left(\Theta_{1}\right), \Psi_{2}\left(Q_{2}\right)\right) w_{k}\left(s, \beta_{2}+1\right), \\
D(s)=\alpha_{12} \mathcal{L}_{1} \mathcal{L}_{2} \sum_{k=1}^{\infty} \pi_{k}\left(\Psi_{1}\left(Q_{1}\right), \Psi_{2}\left(\Theta_{2}\right) w_{k}\left(s, \beta_{2}+1\right),\right.
\end{gathered}
$$

hence the claim follows. 
Proof of Proposition 4.1 For $j \neq i$, we have

$$
\begin{aligned}
\mathbb{E}\left(X_{i} \mathbb{1}_{\left\{S_{2}=s\right\}}\right)= & \int_{0}^{s} y h(y, s-y) d y \\
= & \left(1+\alpha_{12} \mathcal{L}_{i} \mathcal{L}_{j}\right) \int_{0}^{s} y f_{i}(y) f_{j}(s-y) d y \\
& +\alpha_{12} \int_{0}^{s} y e^{-y} f_{i}(y) e^{-(s-y)} f_{j}(s-y) d y-\alpha_{12} \mathcal{L}_{j} \int_{0}^{s} y e^{-y} f_{i}(y) \\
& \times f_{j}(s-y) d y-\alpha_{12} \mathcal{L}_{i} \int_{0}^{s} y f_{i}(y) e^{-(s-y)} f_{j}(s-y) d y .
\end{aligned}
$$

Let $A(s), B(s), C(s), D(s)$ be the four terms of the expression of $\mathbb{E}\left(X_{i} \mathbb{1}_{\left\{S_{2}=s\right\}}\right)$ respectively. In light of Cossette et al. (2013), Lemma 2.5, if $X_{i} \sim M E\left(\beta_{i}, Q_{i}\right)$ then $\frac{x_{i} f_{i}\left(x_{i}, \beta_{i}, Q_{i}\right)}{\mathbb{E}\left(X_{i}\right)}$ can be expressed as a pdf of mixed Erlang distribution with mixing probabilities $G_{i}\left(Q_{i}\right)=\left(g_{1}, g_{2}, \ldots\right)$ where the k-th individual mixing probability is given by

$$
g_{k}=\left\{\begin{array}{lll}
0 & \text { for } & k=1 \\
\frac{(k-1) q_{i, k-1}}{\sum_{j=1}^{k-1} j q_{i, j}} & \text { for } & k>1
\end{array}\right.
$$

If we set $\mu_{i}:=\mathbb{E}\left(X_{i}\right)=\frac{1}{\beta_{i}} \sum_{k=1}^{\infty} k q_{i k}, \gamma:=\alpha_{12} \mathcal{L}_{1} \mathcal{L}_{2}$, then using (7.1), Lemma 5.1, 5.2 and 5.3, one can write $A(s)$ as

$$
A(s)=(1+\gamma) \mu_{i} \sum_{k=1}^{\infty} \pi_{k}\left\{\Psi_{i}\left(G_{i}\left(Q_{i}\right)\right), \Psi_{j}\left(Q_{j}\right)\right\} w_{k}\left(s, \beta_{2}+1\right) .
$$

Setting $\tilde{\mu}_{i}:=\frac{1}{\beta_{i}+1} \sum_{k=1}^{\infty} k \theta_{i k}$, in light of (7.1), Lemma 5.1, 5.2 and 5.3, similarly to $A(s)$, we get the expression of the last three terms of $\mathbb{E}\left(X_{i} \mathbb{1}_{\left\{S_{2}=s\right\}}\right)$ as follows

$$
\begin{aligned}
& B(s)=\gamma \tilde{\mu}_{i} \sum_{k=1}^{\infty} \pi_{k}\left\{\Psi_{i}\left(G_{i}\left(\Theta_{i}\right)\right), \Psi_{j}\left(\Theta_{j}\right)\right\} w_{k}\left(s, \beta_{2}+1\right), \\
& C(s)=-\gamma \tilde{\mu}_{i} \sum_{k=1}^{\infty} \pi_{k}\left\{\Psi_{i}\left(G_{i}\left(\Theta_{i}\right)\right), \Psi_{j}\left(Q_{j}\right)\right\} w_{k}\left(s, \beta_{2}+1\right), \\
& D(s)=-\gamma \mu_{i} \sum_{k=1}^{\infty} \pi_{k}\left\{\Psi_{i}\left(G_{i}\left(Q_{i}\right)\right), \Psi_{j}\left(\Theta_{j}\right)\right\} w_{k}\left(s, \beta_{2}+1\right) .
\end{aligned}
$$


Hence, in view of (4.1)

$$
\operatorname{TVa}_{p}\left(X_{i}, S_{2}\right)=\frac{1}{1-p} \sum_{k=1}^{\infty} z_{i, k} \bar{W}_{k}\left(\operatorname{Va} R_{S_{2}}(p), \beta_{2}+1\right),
$$

where $z_{i k}$ is given in (4.3). Next, by Lemma 5.2, since $\beta_{1} \leqslant \beta_{2}$ we obtain

$$
\begin{aligned}
\mathbb{E}\left(X_{i}\right) & =\frac{1}{\beta_{2}+1} \sum_{k=1}^{\infty} k \psi_{i, k}, \\
\operatorname{Var}\left(X_{i}\right) & =\frac{1}{\left(\beta_{2}+1\right)^{2}}\left(\sum_{m=1}^{\infty}\left(m^{2}+m\right) \psi_{i, m}-\left(\sum_{m=1}^{\infty} m \psi_{i, m}\right)^{2}\right) .
\end{aligned}
$$

In light of (A.1), we know that for $i \neq j$

$$
\begin{aligned}
\operatorname{Cov}\left(X_{i}, X_{j}\right)= & \alpha_{12}\left(\frac{1}{\beta_{i}+1} \sum_{m=1}^{\infty} m q_{i, m}{\overline{\beta_{i}}}^{m}-\sum_{m=1}^{\infty} q_{i, m}{\overline{\beta_{i}}}^{m} \frac{1}{\beta_{i}} \sum_{m=1}^{\infty} m q_{i, m}\right) \\
& \times\left(\frac{1}{\beta_{j}+1} \sum_{s=1}^{\infty} s q_{j, s}{\overline{\beta_{j}}}^{s}-\sum_{s=1}^{\infty} q_{j, s}{\overline{\beta_{j}}}^{s} \frac{1}{\beta_{j}} \sum_{s=1}^{\infty} s q_{j, s}\right),
\end{aligned}
$$

Furthermore, Proposition 3.1 and (2.16) yield

$$
\begin{aligned}
& \mathbb{E}\left(S_{2}\right)=\frac{1}{\beta_{2}+1} \sum_{k=1}^{\infty} k p_{k}, \quad \operatorname{Var}\left(S_{2}\right)=\frac{1}{\left(\beta_{2}+1\right)^{2}}\left(\sum_{m=1}^{\infty}\left(m^{2}+m\right) p_{m}-\left(\sum_{m=1}^{\infty} m p_{m}\right)^{2}\right), \\
& T V a R_{S_{2}}(p)=\frac{1}{\left(\beta_{2}+1\right) \varphi} \sum_{k=0}^{\infty} \frac{P_{k}^{*}\left(\left(\beta_{2}+1\right) V a R_{S_{2}}(p)\right)^{k}}{k !}+\operatorname{VaR}_{S_{2}}(p) .
\end{aligned}
$$

where

$$
\begin{aligned}
\varphi & =\sum_{j=1}^{\infty} \frac{P_{j}\left(\left(\beta_{2}+1\right) V a R_{S_{2}}(l)\right)^{j-1}}{(j-1) !}, \quad P_{k}^{*}=\sum_{j=k}^{\infty} P_{j}, \\
P_{j} & =\frac{\sum_{k=j}^{\infty} p_{k}}{\sum_{k=1}^{\infty} k p_{k}}, \quad \text { and } p_{k} \text { is given in (3.1). }
\end{aligned}
$$

Setting $L_{i, k}:=k \psi_{i, k}+\varepsilon_{i, j}\left(\frac{P_{k}^{*}\left(\left(\beta_{2}+1\right) V a R_{S_{2}}(p)\right)^{k}}{\varphi k !}+\left(\beta_{2}+1\right) V a R_{S_{2}}(p)-k p_{k}\right)$ and plugging the value of $\mathbb{E}\left(X_{i}\right), \operatorname{Var}\left(X_{i}\right), \operatorname{Cov}\left(X_{i}, X_{j}\right), \operatorname{Var}\left(S_{2}\right), T \operatorname{Va} R_{S_{2}}(p)$ and $\mathbb{E}\left(S_{2}\right)$ in (4.2), we obtain the desired result for $K_{p}\left(X_{i}, S_{2}\right)$ where $\varepsilon_{i, j}$ is given in (4.4). 
Proof OF Lemma 5.1 We have

$$
\begin{aligned}
g^{\theta}(x, \beta+1, \Theta) & =\frac{e^{-x} g(x, \beta, Q)}{\mathcal{L}} \\
& =\sum_{k=1}^{\infty} q_{k} \frac{\beta^{k} x^{k-1} e^{-\beta x}}{(k-1) !} \frac{e^{-x}}{\mathcal{L}} \\
& =\sum_{k=1}^{\infty} \frac{q_{k}\left(\frac{\beta}{\beta+1}\right)^{k}}{\sum_{j=1}^{\infty} q_{j}\left(\frac{\beta}{\beta+1}\right)^{j}} w_{k}(x, \beta+1) \\
& =\sum_{k=1}^{\infty} \theta_{k} w_{k}(x, \beta+1) .
\end{aligned}
$$

Proof of Proposition 6.1 By definition

$$
f_{S_{n}}(s)=\int_{0}^{s} \int_{0}^{s-x_{1}} \cdots \int_{0}^{s-x_{1}-\cdots-x_{n-2}} h\left(x_{1}, x_{2}, \ldots, s-x_{1}-\ldots-x_{n-1}\right) d x_{n-1} \ldots d x_{2} d x_{1} .
$$

For $C=\{1, \ldots, n\}$, if we decompose the pdf $h$ in (2.10), we obtain

$$
\begin{aligned}
h(\boldsymbol{x})= & \left(1+\sum_{j_{1}} \sum_{j_{2}} \alpha_{j_{1}, j_{2}} \mathcal{L}_{j_{1}} \mathcal{L}_{j_{2}}-\sum_{j_{1}} \sum_{j_{2}} \sum_{j_{3}} \alpha_{j_{1}, j_{2}, j_{3}} \mathcal{L}_{j_{1}} \mathcal{L}_{j_{2}} \mathcal{L}_{j_{3}}+\cdots\right. \\
& \left.+(-1)^{n} \alpha_{1,2, \ldots, n} \prod_{i=1}^{n} \mathcal{L}_{i}\right) \prod_{i=1}^{n} f_{i}\left(x_{i}\right)+\sum_{j_{1}}\left(-\sum_{j_{2}} \alpha_{j_{1}, j_{2}} \mathcal{L}_{j_{2}}+\sum_{j_{2}} \sum_{j_{3}} \alpha_{j_{1}, j_{2}, j_{3}}\right. \\
& \left.\times \mathcal{L}_{j_{2}} \mathcal{L}_{j_{3}}+\cdots+(-1)^{n+1} \alpha_{1,2, \ldots, n} \prod_{i \in C \backslash\left\{j_{1}\right\}} \mathcal{L}_{i}\right) \tilde{f}_{j_{1}}\left(x_{j_{1}}\right) \prod_{i \in C \backslash\left\{j_{1}\right\}} f_{i}\left(x_{i}\right) \\
& +\sum_{j_{1}} \sum_{j_{2}}\left(\alpha_{j_{1}, j_{2}}-\sum_{j_{3}} \alpha_{j_{1}, j_{2}, j_{3}} \mathcal{L}_{j_{3}}+\sum_{j_{3}} \sum_{j_{4}} \alpha_{j_{1}, j_{2}, j_{3}, j_{4}} \mathcal{L}_{j_{3}} \mathcal{L}_{j_{4}}+\cdots\right. \\
& \left.+(-1)^{n} \alpha_{1,2, \ldots, n} \prod_{i \in C \backslash\left\{j_{1}, j_{2}\right\}} \mathcal{L}_{i}\right) \tilde{f}_{j_{1}}\left(x_{j_{1}}\right) \tilde{f}_{j_{2}}\left(x_{j_{2}}\right) \prod_{i \in C \backslash\left\{j_{1}, j_{2}\right\}} f_{i}\left(x_{i}\right) \\
& +\sum_{j_{1}} \sum_{j_{2}} \sum_{j_{3}}\left(\alpha_{j_{1}, j_{2}, j_{3}}-\sum_{j_{4}} \alpha_{j_{1}, j_{2}, j_{3}, j_{4}} \mathcal{L}_{j_{4}}+\sum_{j_{4}} \sum_{j_{5}} \alpha_{j_{1}, j_{2}, j_{3}, j_{4}, j_{5}} \mathcal{L}_{j_{4}} \mathcal{L}_{j_{5}}\right. \\
& \left.+\cdots+(-1)^{n+1} \alpha_{1,2, \ldots, n} \prod_{i \in C \backslash\left\{j_{1}, j_{2}\right\}} \mathcal{L}_{i}\right) \tilde{f}_{j_{1}}\left(x_{j_{1}}\right) \tilde{f}_{j_{2}}\left(x_{j_{2}}\right) \tilde{f}_{j_{3}}\left(x_{j_{3}}\right) \prod_{\left.i \in C \backslash j_{1}, j_{2}, j_{3}\right\}} f_{i}\left(x_{i}\right)
\end{aligned}
$$




$$
\begin{aligned}
& +\cdots+\sum_{j_{1}} \sum_{j_{2}} \ldots \sum_{j_{n-1}}\left(\alpha_{j_{1}, j_{2}, \ldots, j_{n-1}}-\alpha_{1,2, \ldots, n} \mathcal{L}_{j_{n}}\right) \tilde{f}_{j_{1}}\left(x_{j_{1}}\right) \times \ldots \\
& \times \tilde{f}_{j_{n-1}}\left(x_{j_{n-1}}\right) f_{j_{n}}\left(x_{j_{n}}\right)+\alpha_{1,2, \ldots, n} \prod_{i=1}^{n} \tilde{f}_{i}\left(x_{i}\right),
\end{aligned}
$$

where $j_{1} \in C, j_{2} \in C \backslash\left\{j_{1}\right\}, j_{3} \in C \backslash\left\{j_{1}, j_{2}\right\}, \ldots, j_{n} \in C \backslash\left\{j_{1}, \ldots, j_{n-1}\right\}$. Hence, using (7.3), one can express (7.2) as follows

$$
\begin{aligned}
& f_{S_{n}}(s)=\left(1+\sum_{j_{1}} \sum_{j_{2}} \alpha_{j_{1}, j_{2}} \mathcal{L}_{j_{1}} \mathcal{L}_{j_{2}}-\sum_{j_{1}} \sum_{j_{2}} \sum_{j_{3}} \alpha_{j_{1}, j_{2}, j_{3}} \mathcal{L}_{j_{1}} \mathcal{L}_{j_{2}} \mathcal{L}_{j_{3}}+\cdots\right. \\
& \left.+(-1)^{n} \alpha_{1,2, \ldots, n} \prod_{i=1}^{n} \mathcal{L}_{i}\right) \int_{0}^{s} \int_{0}^{s-x_{1}} \ldots \int_{0}^{s-x_{1}-\ldots-x_{n-2}} \prod_{i=1}^{n-1} f_{i}\left(x_{i}\right) \\
& \times f_{n}\left(s-x_{1}-\ldots-x_{n-1}\right) d x_{n-1} \ldots d x_{2} d x_{1}+\sum_{j_{1}}\left(-\sum_{j_{2}} \alpha_{j_{1}, j_{2}} \mathcal{L}_{j_{2}}\right. \\
& \left.+\sum_{j_{2}} \sum_{j_{3}} \alpha_{j_{1}, j_{2}, j_{3}} \mathcal{L}_{j_{2}} \mathcal{L}_{j_{3}}+\cdots+(-1)^{n+1} \alpha_{1,2, \ldots, n} \prod_{i \in C \backslash\left\{j_{1}\right\}} \mathcal{L}_{i}\right) \\
& \times \int_{0}^{s} \int_{0}^{s-x_{1}} \cdots \int_{0}^{s-x_{1}-\ldots-x_{n-2}} \tilde{f}_{j_{1}}\left(x_{j_{1}}\right) \prod_{i \in C \backslash\left\{j_{1}\right\}} f_{i}\left(x_{i}\right) f_{n}\left(s-x_{1}-\ldots\right. \\
& \left.-x_{n-1}\right) d x_{n-1} \ldots d x_{2} d x_{1}+\sum_{j_{1}} \sum_{j_{2}}\left(\alpha_{j_{1}, j_{2}}-\sum_{j_{3}} \alpha_{j_{1}, j_{2}, j_{3}} \mathcal{L}_{j_{3}}\right. \\
& \left.+\sum_{j_{3}} \sum_{j_{4}} \alpha_{j_{1}, j_{2}, j_{3}, j_{4}} \mathcal{L}_{j_{3}} \mathcal{L}_{j_{4}}+\cdots+(-1)^{n} \alpha_{1,2, \ldots, n} \prod_{i \in C \backslash\left\{j_{1}, j_{2}\right\}} \mathcal{L}_{i}\right) \\
& \times \int_{0}^{s} \int_{0}^{s-x_{1}} \cdots \int_{0}^{s-x_{1}-\ldots-x_{n-2}} \tilde{f}_{j_{1}}\left(x_{j_{1}}\right) \tilde{f}_{j_{2}}\left(x_{j_{2}}\right) \prod_{i \in C \backslash\left\{j_{1}, j_{2}\right\}} f_{i}\left(x_{i}\right) \\
& \times f_{n}\left(s-x_{1}-\ldots-x_{n-1}\right) d x_{n-1} \ldots d x_{2} d x_{1}+\sum_{j_{1}} \sum_{j_{2}} \sum_{j_{3}}\left(\alpha_{j_{1}, j_{2}, j_{3}}\right. \\
& -\sum_{j_{4}} \alpha_{j_{1}, j_{2}, j_{3}, j_{4}} \mathcal{L}_{j_{4}}+\sum_{j_{4}} \sum_{j_{5}} \alpha_{j_{1}, j_{2}, j_{3}, j_{4}, j_{5}} \mathcal{L}_{j_{4}} \mathcal{L}_{j_{5}}+\cdots+(-1)^{n+1} \alpha_{1,2, \ldots, n} \\
& \left.\times \prod_{i \in C \backslash\left\{j_{1}, j_{2}\right\}} \mathcal{L}_{i}\right) \int_{0}^{s} \int_{0}^{s-x_{1}} \cdots \int_{0}^{s-x_{1}-\ldots-x_{n-2}} \tilde{f}_{j_{1}}\left(x_{j_{1}}\right) \tilde{f}_{j_{2}}\left(x_{j_{2}}\right) \tilde{f}_{j_{3}}\left(x_{j_{3}}\right)
\end{aligned}
$$




$$
\begin{aligned}
& \times \prod_{i \in C \backslash\left\{j_{1}, j_{2}, j_{3}\right\}} f_{i}\left(x_{i}\right) f_{n}\left(s-x_{1}-\ldots-x_{n-1}\right) d x_{n-1} \ldots d x_{2} d x_{1}+\ldots \\
& +\sum_{j_{1}} \sum_{j_{2}} \ldots \sum_{j_{n-1}}\left(\alpha_{j_{1}, j_{2}, \ldots, j_{n-1}}-\alpha_{1,2, \ldots, n} \mathcal{L}_{j_{n}}\right) \int_{0}^{s} \int_{0}^{s-x_{1}} \ldots \int_{0}^{s-x_{1}-\ldots-x_{n-2}} \\
& \times \tilde{f}_{1}\left(x_{1}\right) \times \ldots \times \tilde{f}_{j_{n-1}}\left(x_{j n-1}\right) f_{j n}\left(s-x_{1}-\ldots-x_{n-1}\right) d x_{n-1} \ldots d x_{2} d x_{1} \\
& +\alpha_{1,2, \ldots, n} \int_{0}^{s} \int_{0}^{s-x_{1}} \ldots \int_{0}^{s-x_{1}-\ldots-x_{n-2}} \prod_{i=1}^{n-1} \tilde{f}_{i}\left(x_{i}\right) \tilde{f}_{n}\left(s-x_{1}-\ldots\right. \\
& \left.-x_{n-1}\right) d x_{n-1} \ldots d x_{2} d x_{1} .
\end{aligned}
$$

It can be seen that the pdf of $S_{n}$ is a sum of convolutions of mixed Erlang distributions. Thus, as in the case of $S_{2}, S_{n}$ follows a mixed Erlang distribution with scale parameter $\beta_{n}+1$ and mixing probabilities $P=\left(p_{1}, p_{2}, \ldots\right)$, we write $S_{n} \sim M E\left(\beta_{n}+1, P\right)$. For $k \in \mathbb{N}^{*}$, the k-th component $p_{k}$ of $\underset{\sim}{P}$ is given in (6.1).

ProOf Of Proposition 6.3 In view of (4.1) we need to evaluate

$$
\begin{aligned}
\mathbb{E}\left(X_{m} \mathbb{1}_{\left\{S_{n}=s\right\}}\right)= & \int_{0}^{s} \int_{0}^{s-x_{1}} \ldots \int_{0}^{s-x_{1}-\ldots-x_{n-2}} \\
& \times x_{m} h\left(x_{1}, x_{2}, \ldots, s-x_{1}-\ldots-x_{n-1}\right) d x_{n-1} \ldots d x_{2} d x_{1} .
\end{aligned}
$$

If we decompose $x_{m} h(\boldsymbol{x})$, we have

$$
\begin{aligned}
x_{m} h(\boldsymbol{x})= & \left(1+\sum_{j_{1}} \sum_{j_{2}} \alpha_{j_{1}, j_{2}} \mathcal{L}_{j_{1}} \mathcal{L}_{j_{2}}-\sum_{j_{1}} \sum_{j_{2}} \sum_{j_{3}} \alpha_{j_{1}, j_{2}, j_{3}} \mathcal{L}_{j_{1}} \mathcal{L}_{j_{2}} \mathcal{L}_{j_{3}}+\cdots\right. \\
& \left.+(-1)^{n} \alpha_{1,2, \ldots, n} \prod_{i=1}^{n} \mathcal{L}_{i}\right)\left(x_{m} f_{m}\left(x_{m}\right) \prod_{i \neq m} f_{i}\left(x_{i}\right)\right)+\sum_{j_{1} \neq m}\left(-\sum_{j_{2}} \alpha_{j_{1}, j_{2}} \mathcal{L}_{j_{2}}\right. \\
& \left.+\sum_{j_{2}} \sum_{j_{3}} \alpha_{j_{1}, j_{2}, j_{3}} \mathcal{L}_{j_{2}} \mathcal{L}_{j_{3}}+\cdots+(-1)^{n+1} \alpha_{1,2, \ldots, n} \prod_{i \in C \backslash\left\{j_{1}\right\}} \mathcal{L}_{i}\right) \\
& \times\left(x_{m} f_{m}\left(x_{m}\right) \tilde{f}_{j_{1}}\left(x_{j_{1}}\right) \prod_{i \in C \backslash\left\{m, j_{1}\right\}} f_{i}\left(x_{i}\right)\right)+\left(-\sum_{j_{2} \neq m} \alpha_{m, j_{2}} \mathcal{L}_{j_{2}}\right. \\
& \left.+\sum_{j_{2} \neq m} \sum_{j_{3} \neq m} \alpha_{m, j_{2}, j_{3}} \mathcal{L}_{j_{2}} \mathcal{L}_{j_{3}}+\cdots+(-1)^{n+1} \alpha_{1,2, \ldots, n} \prod_{i \in C \backslash\{m\}} \mathcal{L}_{i}\right) \\
& \times\left(x_{m} \tilde{f}_{m}\left(x_{m}\right) \prod_{i \in C \backslash\{m\}} f_{i}\left(x_{i}\right)\right)+\sum_{j_{1} \neq m} \sum_{j_{2}}\left(\alpha_{j_{1}, j_{2}}-\sum_{j_{3}} \alpha_{j_{1}, j_{2}, j_{3}} \mathcal{L}_{j_{3}}\right.
\end{aligned}
$$




$$
\begin{aligned}
& \left.+\sum_{j_{3}} \sum_{j_{4}} \alpha_{j_{1}, j_{2}, j_{3}, j_{4}} \mathcal{L}_{j_{3}} \mathcal{L}_{j_{4}}+\cdots+(-1)^{n} \alpha_{1,2, \ldots, n} \prod_{i \in C \backslash\left\{j_{1}, j_{2}\right\}} \mathcal{L}_{i}\right) \\
& \times\left(x_{m} f_{m}\left(x_{m}\right) \tilde{f}_{j_{1}}\left(x_{j_{1}}\right) \tilde{f}_{j_{2}}\left(x_{j_{2}}\right) \prod_{i \in C \backslash\left\{j_{1}, j_{2}, m\right\}} f_{i}\left(x_{i}\right)\right) \\
& +\sum_{j_{2} \neq m}\left(\alpha_{m, j_{2}}-\sum_{j_{3} \neq m} \alpha_{m, j_{2}, j_{3}} \mathcal{L}_{j_{3}}+\sum_{j_{3} \neq m} \sum_{j_{4} \neq m} \alpha_{m, j_{2}, j_{3}, j_{4}} \mathcal{L}_{j_{3}} \mathcal{L}_{j_{4}}+\cdots\right. \\
& \left.+(-1)^{n} \alpha_{1,2, \ldots, n} \prod_{i \in C \backslash\left\{m, j_{2}\right\}} \mathcal{L}_{i}\right)\left(x_{m} \tilde{f}_{m}\left(x_{m}\right) \tilde{f}_{j_{2}}\left(x_{j_{2}}\right) \prod_{i \in C \backslash\left\{m, j_{2}\right\}} f_{i}\left(x_{i}\right)\right)+\cdots \\
& +\sum_{j_{1} \neq m} \sum_{j_{2}} \ldots \sum_{j_{n-1}}\left(\alpha_{j_{1}, j_{2}, \ldots, j_{n-1}}-\alpha_{1,2, \ldots, n} \mathcal{L}_{m}\right)\left(x_{m} f_{m}\left(x_{m}\right) \prod_{k=1, j_{k} \neq m}^{n} \tilde{f}_{j_{k}}\left(x_{j_{k}}\right)\right) \\
& +\sum_{j_{2} \neq m} \ldots \sum_{j_{n-1} \neq m}\left(\alpha_{m, j_{2}, \ldots, j_{n-1}}-\alpha_{1,2, \ldots, n} \mathcal{L}_{j_{n} \neq m}\right) \\
& \times\left(x_{m} \tilde{f}_{m}\left(x_{m}\right) f_{j_{n}}\left(x_{j_{n}}\right) \prod_{k=1, j_{k} \neq m}^{n-1} \tilde{f}_{j_{k}}\left(x_{j_{k}}\right)\right)+\alpha_{1,2, \ldots, n} x_{m} \tilde{f}_{m}\left(x_{m}\right) \prod_{i \neq m} \tilde{f}_{i}\left(x_{i}\right) .
\end{aligned}
$$

Plugging (7.5) in (7.4) and using (7.1), Lemma 5.1, 5.2, and 5.3, similarly to the bivariate case one may express (7.4) as follows

$$
\mathbb{E}\left(X_{m} \mathbb{1}_{\left\{S_{n}=s\right\}}\right)=\sum_{k=1}^{\infty} z_{m, k} \bar{W}_{k}\left(\operatorname{VaR}_{S_{n}}(p), \beta_{n}+1\right),
$$

where $z_{m, k}$ is given in (6.2). Hence, the proof follows easily.

\section{ACKNOWLEDGEMENTS}

E. Hashorva is partially supported by the Swiss National Science Foundation grants 200021-13478, 200021-140633/1. G. Ratovomirija is partially supported by the project RARE -318984 (an FP7 Marie Curie IRSES Fellowship) and Vaudoise Assurances.

\section{REFERENCES}

BAIRAmov, I., Kotz, S. and BeKÇI, M. (2001) New generalized Farlie-Gumbel-Morgenstern distributions and concomitants of order statistics. Journal of Applied Statistics, 28(5), 521-536. 
Cossette, H., M.-P. Côté, Marceau, E. and Moutanabbir, K. (2013) Multivariate distribution defined with Farlie-Gumbel-Morgenstern copula and mixed Erlang marginals: Aggregation and capital allocation. Insurance: Mathematics and Economics, 52, 560-572.

Cossette, H., Mailhot, M. and Marceau, E. (2012) TVaR-based capital allocation for multivariate compound distributions with positive continuous claim amounts. Insurance: Mathematics and Economics, 50(2), 247-256.

CUmmins, J.D. (2000) Allocation of capital in the insurance industry. Risk Management and Insurance Review, 3(1) 7-27.

Denuit, M., Dhaene, J., Goovaerts, M. and KaAs, R. (2005) Actuarial Theory for Dependent Risks: Measures, Orders and Models, Wiley.

DhaEne, J., TSANAKAS, A., VALDEZ, E.A. and VANDUfFEL, S. (2012) Optimal capital allocation principles. Journal of Risk and Insurance, 79(1), 1-28.

DiCKSON, D.C.M. (2008) Some explicit solutions for the joint density of the time of ruin and the deficit at ruin. ASTIN Bulletin, 38(1), 259-276.

Dickson, D.C.M. and Willmot, G.E. (2005) The density of the time to ruin in the classical poisson risk model. ASTIN Bulletin, 35(1), 45-60.

Embrechts, P., Hashorva, E. and Mikosch, T. (2014) Aggregation of log-linear risks. Journal Applied Probability, in press.

FARLIE, D.J.G. (1960) The performance of some correlation coefficients for a general bivariate distribution. Biometrika, 47, 307-323.

Gumbel, E.J. (1960) Bivariate exponential distributions. Journal of the American Statistical Association, 55, 698-707.

HASHORVA, E. and KORTSCHAK D. (2014) Tail asymptotics of random sum and maximum of log-normal risks. Statistics Probability Letters, 87, 167-174.

HASHORVA, E. and LI, J. (2015) Tail behaviour of weighted sums of order statistics of dependent risks. Stochastic Models, in press, 31(1)-.

HERnÁndeZ-BASTIDA, A. and FERnÁNDEZ-SÁnCHEZ, M.P. (2012) A sarmanov family with beta and gamma marginal distributions: an application to the Bayes premium in a collective risk model. Statistical Methods \& Applications, 21(4), 391-409.

JoE, H. Multivariate Models and Multivariate Dependence Concepts. (1997) Chapman \& Hall/CRC Monographs on Statistics \& Applied Probability. Taylor \& Francis.

Klugman, S.A., Panjer, H.H. and Willmot G.E. (2008) Loss models: From data to decisions, 3rd ed. Wiley Series in Probability and Statistics. Hoboken, NJ: John Wiley \& Sons Inc.

LEE, M.L.T. (1996) Properties and applications of the sarmanov family of bivariate distributions. Communications in Statistics-Theory and Methods, 25(6), 1207-1222.

LEE, S.C.K. and LIN, X.S. (2010) Modeling and evaluating insurance losses via mixtures of Erlang distributions. North American Actuarial Journal, 14(1), 107-130.

LEE, S.C.K. and LIN, X.S. (2012) Modeling dependent risks with multivariate Erlang mixtures. ASTIN Bulletin, 42(1), 153-180.

MCNeIL, A.J., Frey, R. and EMBRECHTS, P. (2005) Quantitative risk management: Concepts, Techniques and Tools. Princeton Series in Finance. Princeton, NJ: Princeton University Press.

MORGENSTERN, D. (1956) Einfache Beispiele zweidimensionaler Verteilungen. Mitteilingsblatt für Mathematische Statistik, 8 234-235.

Nelsen, R.B. (1999) An Introduction to Copulas, volume 139 of Lecture Notes in Statistics. New York: Springer-Verlag.

RosenblatT, M. (1952) Remarks on a multivariate transformation. Annals of Mathematical Statistics, 23, 470-472.

SARABIA, J.M. and GómEZ-DÉNIZ, E. (2011) Multivariate Poisson-Beta distributions with applications. Communications in Statistics-Theory and Methods, 40(6), 1093-1108.

SARMANOV, O.V. (1966) Generalized normal correlation and two-dimensional Fréchet classes. Doklady Akademii Nauk SSSR, 168, 32-35.

Tasche, D. Allocating portfolio economic capital to sub-portfolios. Economic Capital: A Practitioner's Guide, Risk Books, pages 275-302, 2004.

Willmot, G.E. and Lin, X.S. (2011) Risk modeling with the mixed Erlang distribution. Applied Stochastic Models in Business and Industry, 27(1), 8-22. 
WiLlmot, G.E. and WoO, J.K. (2007) On the class of Erlang mixtures with risk theoretic applications. North American Actuarial Journal, 11(2), 99-115.

YANG, Y. and HASHORVA, E. (2013) Extremes and products of multivariate AC-product risks. Insurance: Mathematics and Economics, 52(2), 312-319.

YANG, Y. and WANG, Y. (2013) Tail behavior of the product of two dependent random variables with applications to risk theory. Extremes, 16(1), 55-74.

ENKELEJD HASHORVA

Department of Actuarial Science,

Faculty of Business and Economics,

University of Lausanne,

UNIL-Dorigny 1015 Lausanne, Switzerland

E-Mail address: Enkelejd.Hashorva@unil.ch

Gildas RATOVOMIRIJA (Corresponding author)

Department of Actuarial Science,

Faculty of Business and Economics,

University of Lausanne,

UNIL-Dorigny 1015 Lausanne, Switzerland

E-Mail address: Gildas.Ratovomirija@unil.ch,gra@vaudoise.ch

\section{APPENDIX A}

\section{A. DEPENDENCE MEASURES}

Pearson's correlation coefficient has been widely used as a measure of the dependence between two random variables (rv) $X_{1}$ and $X_{2}$. In this respect, the concept of dependence is assumed to be the linear relationship between the two rv. However, in practice the dependence structure is not always linear hence is why the concept of concordance has been introduced, see e.g., Nelsen (1999), McNeil et al. 2005, or Denuit et al. (2005). By definition, a rv $X_{1}$ is concordant with a rv $X_{2}$ if they tend to vary together. The two measures of association of $X_{1}$ and $X_{2}$, namely Spearman's rho and Kendall's tau are based on this concept. Probabilistically speaking, if $\left(Y_{1}, Y_{2}\right)$ and $\left(Z_{1}, Z_{2}\right)$ are independent copies of the pair of continuous random variables $\left(X_{1}, X_{2}\right)$, then Kendall's tau is defined as

$$
\tau\left(X_{1}, X_{2}\right)=\mathbb{P}\left\{\left(X_{1}-Y_{1}\right)\left(X_{2}-Y_{2}\right)>0\right\}-\mathbb{P}\left\{\left(X_{1}-Y_{1}\right)\left(X_{2}-Y_{2}\right)<0\right\},
$$

and Spearman's rho is defined as

$$
\rho_{S}\left(X_{1}, X_{2}\right)=3\left\{\mathbb{P}\left[\left(X_{1}-Y_{1}\right)\left(X_{2}-Z_{2}\right)>0\right]-\mathbb{P}\left[\left(X_{1}-Y_{1}\right)\left(X_{2}-Z_{2}\right)<0\right]\right\},
$$

where $Y_{1}$ and $Z_{2}$ are independent. If $\left(X_{1}, X_{2}\right) \sim S M E_{2}\left(\boldsymbol{\beta}, Q_{1}, Q_{2}\right)$ and further $X_{i}, i=1,2$ has finite mean, then we have: 
TABLE A.1

MIXTURE PARAMETERS OF MARGINALS.

\begin{tabular}{rcrrr}
\hline \hline \multicolumn{2}{c}{$X_{1}$} & & \multicolumn{2}{c}{$X_{2}$} \\
\cline { 5 - 5 }$k$ & $q_{1, k}$ & & $k$ & $q_{2, k}$ \\
\hline 1 & 0.5270 & & 1 & 0.5050 \\
40 & 0.0005 & & 8 & 0.0150 \\
50 & 0.0020 & & 30 & 0.0105 \\
75 & 0.0010 & & 50 & 0.0020 \\
150 & 0.0015 & & 70 & 0.0015 \\
345 & 0.0005 & & 95 & 0.0010 \\
902 & 0.0050 & & 850 & 0.0055 \\
970 & 0.4375 & & 995 & 0.1050 \\
993 & 0.0250 & & 1000 & 0.3545 \\
\hline \hline
\end{tabular}

\section{Pearson's correlation coefficient:}

If we set $\eta_{i k}:=\frac{1}{\beta_{i}+1} \sum_{k=1}^{\infty} k q_{i, k} \bar{\beta}_{i}^{k}$ and $\Gamma_{i k}:=\sum_{k=1}^{\infty} q_{i, k} \bar{\beta}_{i}^{k} \mu_{i}$ for $i=1,2$, then by (2.6) Pearson's correlation coefficient of the bivariate SmE risks has an explicit form as

$$
\rho_{12}\left(X_{1}, X_{2}\right)=\frac{\alpha_{12}\left(\eta_{1, k}-\Gamma_{1, k}\right)\left(\eta_{2, k}-\Gamma_{2, k}\right)}{\sigma_{1} \sigma_{2}},
$$

where $\mu_{i}$ is the expected value of $X_{i}, i=1,2$ and $\sigma_{i}$ is its standard deviation.

Remarks A.1. According to (2.7), the maximal value of Pearson's correlation coefficient of the bivariate SmE risks can be written as follows

$$
\rho_{12}^{\max }\left(X_{1}, X_{2}\right)=\frac{\left(\eta_{1, k}-\Gamma_{1, k}\right)\left(\eta_{2, k}-\Gamma_{2, k}\right)}{\max \left\{\mathcal{L}_{1}\left(1-\mathcal{L}_{2}\right),\left(1-\mathcal{L}_{1}\right) \mathcal{L}_{2}\right\} \sigma_{1} \sigma_{2}}
$$

and its minimal value can be expressed as

$$
\rho_{12}^{\min }\left(X_{1}, X_{2}\right)=\frac{-\left(\eta_{1, k}-\Gamma_{1, k}\right)\left(\eta_{2, k}-\Gamma_{2, k}\right)}{\max \left\{\mathcal{L}_{1} \mathcal{L}_{2},\left(1-\mathcal{L}_{1}\right)\left(1-\mathcal{L}_{2}\right)\right\} \sigma_{1} \sigma_{2}} .
$$

In the following example, we show that the SmE distribution is flexible as a model for dependent risks.

\section{Example A.2. Extremal dependence}

In this example, we analyse the bounds of Pearson's correlation coefficient of a bivariate mixed Erlang distribution with marginals which share the same scale parameter and consist of 9 Erlang components. The mixture parameters are summarized in Table A.1. Figure A.1 presents the lower and the upper bound of Pearson's correlation coefficient as a function of the common scale parameter $\beta$. We can see that $\rho_{12}^{\max }$ and $\rho_{12}^{\min }$ tend to reach the extremal dependence case which correspond to values of 1 and -1 respectively. The strongest negative correlation $\rho_{12}^{\min }=-0.87545$ is attained for $\beta=21.5723$ while the value of $\beta=153.0315$ yields the maximal positive correlation $\rho_{12}^{\max }=0.96871$. Hence, not only is the range of the dependence flexible but also wide. Moreover, the simulated values of $\rho_{12}^{\max }$ and $\rho_{12}^{\min }$, presented in dotted red lines in Figure A.1, correspond well with the exact values, this demonstrates again the robustness of our results. 


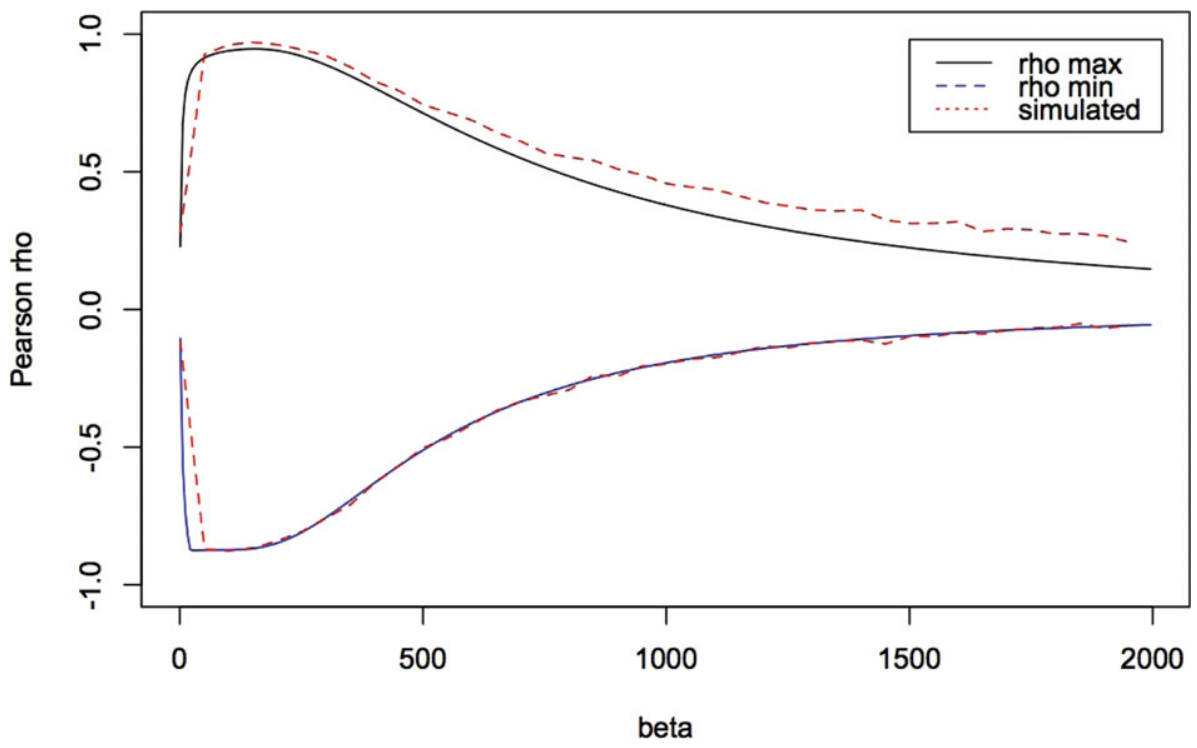

FIGURE A.1: $\rho_{12}^{\max }$ and $\rho_{12}^{\min }$ as a function the common scale parameter $\beta$. (Color online)

2. Spearman's Rho: Spearman's rho of the bivariate SmE risks can be expressed explicitly as follows

$$
\rho_{S}\left(X_{1}, X_{2}\right)=3(1+\gamma)+6 \alpha_{12}\left[2 \zeta_{1} \zeta_{2}-\mathcal{L}_{1} \zeta_{2}-\mathcal{L}_{2} \zeta_{1}\right]-3
$$

where $\zeta_{i}=\sum_{k=1}^{\infty} q_{i, k} \bar{\beta}_{i}^{k} \sum_{m=1}^{\infty} \sum_{j=0}^{k-1} q_{i, m}\left(\begin{array}{c}j+m-1 \\ m-1\end{array}\right) \frac{\beta_{i}^{m}\left(\beta_{i}+1\right)^{j}}{(2 \beta+1)^{m+j}}$, for $i=1,2$.

3. Kendall's Tau: Kendall's tau of the bivariate $\mathrm{SmE}$ is given by the following closed formula

$$
\tau\left(X_{1}, X_{2}\right)=4\left[(1+\gamma) 12\left(\rho_{S}\left(X_{1}, X_{2}\right)+3\right)+\alpha_{12} \tau_{1}-\alpha_{12} \mathcal{L}_{2} \tau_{2}-\alpha_{12} \mathcal{L}_{1} \tau_{3}\right]-1,
$$

where $\rho_{S}\left(X_{1}, X_{2}\right)$ is Spearman's rho,

$$
\begin{aligned}
\tau_{1} & =(1+\gamma) Z_{1} Z_{2}+\alpha_{12} T_{1} T_{2}-\alpha_{12} \mathcal{L}_{1} Z_{1} T_{2}-\alpha_{12} \mathcal{L}_{2} Z_{2} T_{1}, \\
\tau_{2} & =\frac{1}{2}(1+\gamma) Z_{1}+\alpha_{12} T_{1} \zeta_{2}-\alpha_{12} \mathcal{L}_{1} Z_{1} \zeta_{2}-\frac{1}{2} \alpha_{12} \mathcal{L}_{2} T_{1}, \\
\tau_{3} & =\frac{1}{2}(1+\gamma) Z_{2}+\alpha_{12} \zeta_{1} T_{2}-\alpha_{12} \mathcal{L}_{2} \zeta_{1} Z_{2}-\frac{1}{2} \alpha_{12} \mathcal{L}_{1} T_{2},
\end{aligned}
$$

with

$$
\begin{aligned}
& Z_{i}=\sum_{k=1}^{\infty} q_{i, k} \sum_{m=1}^{\infty} \sum_{j=0}^{k-1} q_{i, m}\left(\begin{array}{c}
j+m-1 \\
m-1
\end{array}\right)\left(\frac{\beta_{i}}{2 \beta_{i}+1}\right)^{m+j}, \quad \text { for } \quad i=1,2, \\
& T_{i}=\sum_{k=1}^{\infty} q_{i, k} \bar{\beta}_{i}^{k} \sum_{m=1}^{\infty} \sum_{j=0}^{k-1} q_{i, m}\left(\begin{array}{c}
j+m-1 \\
m-1
\end{array}\right) \frac{\beta_{i}^{m}\left(\beta_{i}+2\right)^{j}}{\left(2 \beta_{i}+2\right)^{m+j}}, \quad \text { for } \quad i=1,2 .
\end{aligned}
$$




\section{B. SIMULATION OF SME RISKS}

In simulation, in order to remove the dependence between two risks $X_{1}$ and $X_{2}$, the Rosenblatt transform introduced by Rosenblatt (1952) is widely used. In fact, to simulate $X_{2}$ this approach consists in using the conditional quantile function of $X_{2}$ given the value of $X_{1}$. Hence, the conditional df of $X_{2}$ is found accordingly. The following lemma yields how this can be done for the case of the bivariate $\mathrm{SmE}$ distribution.

Lemma B.1. Let $\left(X_{1}, X_{2}\right) \sim S M E_{2}\left(\boldsymbol{\beta}, Q_{1}, Q_{2}\right)$, for a given value of $X_{1}$ the conditional df of $X_{2}$ is described as follows

$$
F_{2 \mid 1}\left(x_{2} \mid x_{1}\right)=\lambda F_{2}\left(x_{2}, \beta_{2}, Q_{2}\right)+\alpha_{12} \Delta_{1} \sum_{k=1}^{\infty} q_{2, k} \bar{\beta}_{2}^{k} W_{k}\left(x_{2}, \beta_{2}+1\right),
$$

where

$$
\lambda=1+\alpha_{12} \mathcal{L}_{2}\left(\mathcal{L}_{1}-e^{-x 1}\right), \quad \Delta_{1}=\left(e^{-x 1}-\mathcal{L}_{1}\right) .
$$

Proof. For a given value of $X_{1}$, one can define the conditional distribution function of $X_{2}$ as

$$
F_{2 \mid 1}\left(x_{2} \mid x_{1}\right)=\frac{\int_{0}^{x_{2}} h\left(x_{1}, s\right) d s}{f_{1}\left(x_{1}\right)} .
$$

According to (2.1)

$$
\begin{aligned}
h\left(x_{1}, s\right)= & \left(1+\alpha_{12} \mathcal{L}_{1} \mathcal{L}_{2}\right) f_{1}\left(x_{1}\right) f_{2}(s)+\alpha_{12} e^{-x_{1}} f_{1}\left(x_{1}\right) e^{-s} f_{2}(s) \\
& -\alpha_{12} \mathcal{L}_{2} e^{-x_{1}} f_{1}\left(x_{1}\right) f_{2}(s)-\alpha_{12} \mathcal{L}_{1} e^{-s} f_{2}(s) f_{1}\left(x_{1}\right) \\
= & \left(1+\alpha_{12} \mathcal{L}_{1} \mathcal{L}_{2}-\alpha_{12} \mathcal{L}_{2} e^{-x_{1}}\right) f_{1}\left(x_{1}\right) f_{2}(s) \\
& +\alpha_{12}\left(e^{-x_{1}}-\mathcal{L}_{1}\right) f_{1}\left(x_{1}\right) e^{-s} f_{2}(s) .
\end{aligned}
$$

Setting

$$
\lambda:=1+\alpha_{12} \mathcal{L}_{2}\left(\mathcal{L}_{1}-e^{-x 1}\right) \text { and } \Delta_{1}:=e^{-x 1}-\mathcal{L}_{1},
$$

the expression of $h\left(x_{1}, s\right)$ becomes

$$
h\left(x_{1}, s\right)=\lambda f_{1}\left(x_{1}\right) f_{2}(s)+\alpha_{12} \Delta_{1} f_{1}\left(x_{1}\right) e^{-s} f_{2}(s) .
$$

Hence

$$
\begin{aligned}
F_{2 \mid 1}\left(x_{2} \mid x_{1}\right) & =\frac{\int_{0}^{x_{2}} \lambda f_{1}\left(x_{1}\right) f_{2}(s)+\alpha_{12} \Delta_{1} f_{1}\left(x_{1}\right) e^{-s} f_{2}(s) d s}{f_{1}\left(x_{1}\right)} \\
& =\lambda \int_{0}^{x_{2}} f_{2}(s) d s+\alpha_{12} \Delta_{1} \int_{0}^{x_{2}} e^{-s} f_{2}(s) d s \\
& =\lambda F_{2}\left(x_{2}, \beta_{2}, Q_{2}\right)+\alpha_{12} \Delta_{1} \int_{0}^{x_{2}} e^{-s} \sum_{k=1}^{\infty} q_{2 k} \frac{\beta_{2}^{k}}{(k-1) !} s^{k-1} e^{-\beta_{2} s} d s \\
& =\lambda F_{2}\left(x_{2}, \beta_{2}, Q_{2}\right)+\alpha_{12} \Delta_{1} \sum_{k=1}^{\infty} q_{2 k}\left(\frac{\beta_{2}}{\beta_{2}+1}\right)^{k} W_{k}\left(x_{2}, \beta_{2}+1\right) .
\end{aligned}
$$


The inverse of $F_{2 \mid 1}$ can be computed numerically and as a result the Rosenblatt transform can be implemented efficiently. The simulation algorithm can be summarised as follows:

1. simulate two independent $\operatorname{rv} u_{1}$ and $u_{2}$ uniformly distributed

2. simulate $X_{1}$ using the inverse transform: $x_{1}=F_{1}^{-1}\left(u_{1}\right)$

3. simulate $X_{2}$ using the Rosenblatt transform: $x_{2}=F_{2 \mid 1}^{-1}\left(u_{2} \mid x_{1}\right)$

4. simulate the aggregate rv $S_{2}=X_{1}+X_{2}$.

Remarks B.2. The result in Lemma B.1 can be generalized for the multivariate case. Specifically, if $\left(X_{1}, \ldots, X_{n}\right)$ has a multivariate $\operatorname{SmE}$ distribution with $X_{i} \sim \operatorname{ME}\left(\beta_{i}, Q_{i}\right), i=1, \ldots, n$, for given values of $X_{1}, \ldots, X_{n-1}$ one can express the conditional distribution of $X_{n}$ as follows $($ set $C:=\{1, \ldots, n\})$

$$
F_{n \mid 1, \ldots, n-1}\left(x_{n} \mid x_{1}, \ldots, x_{n-1}\right)=\lambda F_{n}\left(x_{n}, \beta_{n}, Q_{n}\right)+\Delta \sum_{k=1}^{\infty} q_{n, k} \bar{\beta}_{n}^{k} W_{k}\left(x_{n}, \beta_{n}+1\right),
$$

where

$$
\begin{aligned}
& \lambda=\frac{1}{D\left(x_{1}, \ldots, x_{n-1}\right)}\left\{(1+\gamma)+\sum_{j_{1} \neq n}\left(-\sum_{j_{2}} \alpha_{j_{1}, j_{2}} \mathcal{L}_{j_{2}}+\sum_{j_{2}} \sum_{j_{3}} \alpha_{j_{1}, j_{2}, j_{3}} \mathcal{L}_{j_{2}} \mathcal{L}_{j_{3}}\right.\right. \\
& \left.+\cdots+(-1)^{n+1} \alpha_{1,2, \ldots, n} \prod_{i \in C \backslash\left\{j_{1}\right\}} \mathcal{L}_{i}\right) e^{-x_{j_{1}}} \\
& +\sum_{j_{1} \neq n} \sum_{j_{2} \neq n}\left(\alpha_{j_{1}, j_{2}}-\sum_{j_{3}} \alpha_{j_{1}, j_{2}, j_{3}} \mathcal{L}_{j_{3}}+\sum_{j_{3}} \sum_{j_{4}} \alpha_{j_{1}, j_{2}, j_{3}, j_{4}} \mathcal{L}_{j_{3}} \mathcal{L}_{j_{4}}\right. \\
& \left.+\cdots+(-1)^{n} \alpha_{1,2, \ldots, n} \prod_{i \in C \backslash\left\{j_{1}, j_{2}\right\}} \mathcal{L}_{i}\right) e^{-x_{j_{1}}-x_{j_{2}}} \\
& \left.+\cdots+\left(\alpha_{1,2, \ldots, n-1}-\alpha_{1,2, \ldots, n} \mathcal{L}_{n}\right) e^{-x_{1}-\ldots-x_{n-1}}\right\}, \\
& \Delta=\frac{1}{D\left(x_{1}, \ldots, x_{n-1}\right)}\left\{\left(-\sum_{j_{2} \neq n} \alpha_{j_{2}, n} \mathcal{L}_{j_{2}}+\sum_{j_{2} \neq n} \sum_{j_{3} \neq n} \alpha_{j_{2}, j_{3}, n} \mathcal{L}_{j_{2}} \mathcal{L}_{j_{3}}+\cdots+(-1)^{n+1}\right.\right. \\
& \left.\times \alpha_{1,2, \ldots, n} \prod_{i \in C \backslash\{n\}} \mathcal{L}_{i}\right)+\sum_{j_{2} \neq n}\left(\alpha_{j_{2}, n}-\sum_{j_{3} \neq n} \alpha_{j_{2}, j_{3}, n} \mathcal{L}_{j_{3}}+\sum_{j_{3} \neq n} \sum_{j_{4} \neq n} \alpha_{j_{2}, j_{3}, j_{4}, n} \mathcal{L}_{j_{3}} \mathcal{L}_{j_{4}}+\cdots\right. \\
& \left.+(-1)^{n} \alpha_{1,2, \ldots, n} \prod_{i \in C \backslash\left\{j_{1}, n\right\}} \mathcal{L}_{i}\right) e^{-x_{j_{2}}}+\cdots+\sum_{j_{1} \neq n} \sum_{j_{2} \neq n} \ldots \sum_{j_{n-1} \neq n}\left(\alpha_{j_{1}, \ldots, j_{n-1}}-\alpha_{1, \ldots, n}\right. \\
& \left.\left.\times \mathcal{L}_{l, l \in C \backslash\left\{j_{1} \ldots, j_{n-1}\right\}}\right) e^{-x_{j_{1}}-\ldots-x_{j_{n-2}}}+\alpha_{1,2, \ldots, n} e^{-x_{1}}-\ldots-x_{n-1}\right\},
\end{aligned}
$$


with

$$
\begin{aligned}
& D\left(x_{1}, \ldots, x_{n-1}\right)=\left(1+\sum_{j_{1} \neq n} \sum_{j_{2} \neq n} \alpha_{j_{1}, j_{2}}\left(e^{-x_{j_{1}}}-\mathcal{L}_{j_{1}}\right)\left(e^{-x_{j_{2}}}-\mathcal{L}_{j_{2}}\right)+\sum_{j_{1} \neq n} \sum_{j_{2} \neq n} \sum_{j_{3} \neq n} \alpha_{j_{1}, j_{2}, j_{3}}\right. \\
&\left.\times\left(e^{-x_{j_{1}}}-\mathcal{L}_{j_{1}}\right)\left(e^{-x_{j_{2}}}-\mathcal{L}_{j_{2}}\right)\left(e^{-x_{j_{3}}}-\mathcal{L}_{j_{3}}\right)+\cdots+\alpha_{1,2, \ldots, n-1} \prod_{i=1}^{n-1}\left(e^{-x_{i}}-\mathcal{L}_{i}\right)\right), \\
& \gamma=\sum_{j_{1}} \sum_{j_{2}} \alpha_{j_{1}, j_{2}} \mathcal{L}_{j_{1}} \mathcal{L}_{j_{2}}-\sum_{j_{1}} \sum_{j_{2}} \sum_{j_{3}} \alpha_{j_{1}, j_{2}, j_{3}} \mathcal{L}_{j_{1}} \mathcal{L}_{j_{2}} \mathcal{L}_{j_{3}}+\cdots+(-1)^{n} \alpha_{1,2, \ldots, n} \prod_{i=1}^{n} \mathcal{L}_{i},
\end{aligned}
$$

$j_{1} \in C, j_{2} \in C \backslash\left\{j_{1}\right\}, j_{3} \in C \backslash\left\{j_{1}, j_{2}\right\}, \ldots, j_{n} \in C \backslash\left\{j_{1}, \ldots, j_{n-1}\right\}$.

Similarily to the simulation of two dependent SmE risks, one can simulate $n$ dependent SmE risks iteratively. 